ALEA, Lat. Am. J. Probab. Math. Stat. 15, 1311-1334 (2018)

DOI: $10.30757 /$ ALEA.v15-49

\title{
Transition to Shocks in TASEP and Decoupling of Last Passage Times
}

\section{Peter Nejjar}

IST Austria

Am Campus 1,

Klosterneuburg, Lower Austria, Austria.

E-mail address: peter.nejjar@ist.ac.at

\begin{abstract}
We consider the totally asymmetric simple exclusion process in a critical scaling parametrized by $a \geq 0$, which creates a shock in the particle density of order $a T^{-1 / 3}, T$ the observation time. When at $a=0$ one has step initial data, we provide bounds on the limiting law of particle positions for $a>0$, which in particular imply that in the double limit $\lim _{a \rightarrow \infty} \lim _{T \rightarrow \infty}$ one recovers the product limit law and the degeneration of the correlation length observed at shocks of order 1 . This result can be phrased in terms of a general last passage percolation model. We also obtain bounds on the decoupling of two-point functions of several Airy processes.
\end{abstract}

\section{Introduction}

We consider the totally asymmetric simple exclusion process (TASEP). In this model, particles move on $\mathbb{Z}$ and jump one step to the right with rate 1 , subject to the exclusion constraint that there is at most one particle on each site such that particles attempting to jump to an occupied site stay put. A particle configuration at time $T \geq 0$ can be encoded by an $\eta_{T} \in\{0,1\}^{\mathbb{Z}}\left(\eta_{T}(i)=1\right.$ if $i$ is occupied at time $T, \eta_{T}(i)=0$ if not) and $\left(\eta_{T}\right)_{T \geq 0}$ is the TASEP, see Liggett (1985) for its rigorous construction.

Given an initial configuration $\eta_{0}$ in TASEP, let us attach a label $n \in \mathbb{Z}$ to each particle and denote by $x_{n}(T)$ the position of particle $n$ at time $T \geq 0$. Depending on $\eta_{0}$, one has different large time densities of particles $\rho(\xi)$, where, informally, $\rho(\xi)$ is the probability that there is a particle at $\lfloor\xi T\rfloor$ for $T$ large. Formally, $\rho$ is the density function of the measure to which the rescaled empirical particle density converges vaguely as $T \rightarrow \infty$ :

Received by the editors March 27th, 2018; accepted October 8th, 2018.

2010 Mathematics Subject Classification. 60K35.

Key words and phrases. KPZ Universality, TASEP, Shocks, Last Passage Percolation.

Research supported by ERC Advanced Grant No. 338804 and ERC Starting Grant No. 716117. 


$$
\lim _{T \rightarrow \infty} \frac{1}{T} \sum_{i \in \mathbb{Z}} \delta_{\frac{i}{T}} \eta_{T}(i)=\rho(\xi) \mathrm{d} \xi
$$

with $\delta_{x}$ the Dirac measure, and $\rho$ is the unique entropy solution to the Burgers equation. Consider for instance the initial data

$$
x_{n}(0)= \begin{cases}-n, & \text { for }-\left\lfloor a T^{2 / 3}\right\rfloor \leq n \leq 0 \\ -n-\left\lfloor a T^{2 / 3}\right\rfloor & \text { for } n \geq 1,\end{cases}
$$

where $a \geq 0$ is a constant. The density profile created by this initial data does not depend on $a$ and is given in Figure 1.1 left: It has a region where the particle density is linearly decreasing, which is called a rarefaction fan.
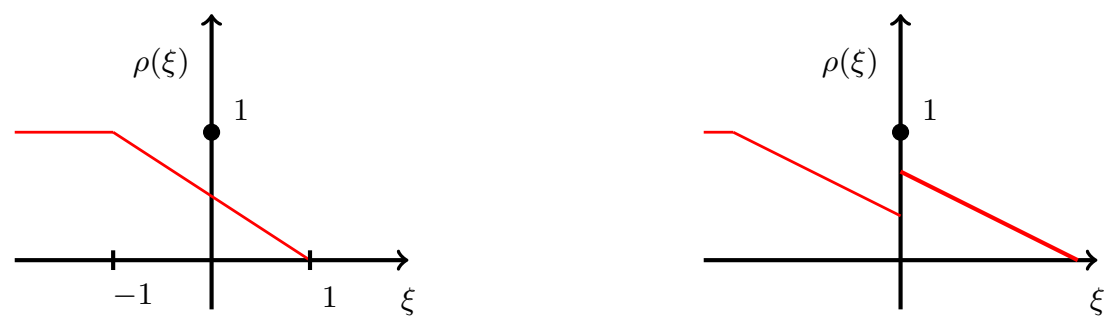

Figure 1.1. Left: Large time density of TASEP started from initial data (1.2). The density $\rho$ decreases linearly from 1 to 0 in the interval $[-1,1]$. Right: Density profile for TASEP started from initial data (1.3). At the origin, two regions of decreasing density come together and the density $\rho$ jumps from $(1-\beta) / 2$ to $(1+\beta) / 2$, i.e. there is a shock.

Consider now in contrast for $\beta \in(0,1)$ the initial configuration

$$
\tilde{x}_{n}(0)= \begin{cases}-n, & \text { for }-\lfloor\beta T\rfloor \leq n \leq 0 \\ -n-\lfloor\beta T\rfloor & \text { for } n \geq 1 .\end{cases}
$$

The density profile has two distinct regions where the density is decreasing and there is a discontinuity between them, see Figure 1.1 right. This is called a shock, which in this case is located at the origin.

The main topic of this paper is the transition of fluctuations of particle positions when $\rho$ is continuous to when $\rho$ has a shock. The fluctuations in these two situations are known to be very different (see (1.11), (1.13) below for the precise statements):

If we choose $\nu>0$ so that $\tilde{x}_{\nu T}(T)$ is located at the shock, i.e. at the origin, the fluctuations of $\tilde{x}_{\nu T}(T)$ are given by a product of two Tracy-Widom $F_{\mathrm{GUE}}$ distributions and particles are non-trivially correlated on the $T^{1 / 3}$ length scale. For $x_{\nu(a) T}(T)$ (where $\nu(a)$ is chosen so that $x_{\nu(a) T}(T)$ is located at the origin, see (1.7)) and $a=0$, however, the fluctuations are given by a single Tracy-Widom $F_{\mathrm{GUE}}$ distribution and particles are non-trivially correlated on the $T^{2 / 3}$ scale.

If we - illegally - set $a=\beta T^{1 / 3}$ (since $a, \beta$ are fixed constants independent of $T$, one cannot have $a=\beta T^{1 / 3}$ ), then $x_{n}$ and $\tilde{x}_{n}$ coincide and (trivially) have the same fluctuation behavior. Continuing with this informal heuristics, letting $T \rightarrow \infty$ in $a=\beta T^{1 / 3}$ leads to $a \rightarrow \infty$ (for $\beta$ fixed) as well as to $\beta \rightarrow 0$ (for $a$ fixed). So if we 
want the two fluctuation behaviors to coincide, it seems reasonable to consider the double limit $\lim _{a \rightarrow \infty} \lim _{T \rightarrow \infty}$ of $x_{\nu(a) T}(T)$, and the double limit $\lim _{\beta \rightarrow 0} \lim _{T \rightarrow \infty}$ of $\tilde{x}_{\nu T}(T)$. As the following corollary of our main result, Theorem 2.1, shows, it is indeed with these two double limits that a continuous transition between the two scaling regimes occurs:

Corollary 1.1. Consider the initial data $x_{n}, \tilde{x}_{n}$ from $(1.2)$, (1.3) and let $u \in \mathbb{R}, \xi=$ $\frac{u}{2} \frac{\beta-1}{\beta}$. Then

$$
\begin{aligned}
& \lim _{a \rightarrow \infty} \lim _{T \rightarrow \infty} \mathbb{P}\left(x_{\left\lfloor\frac{T}{4}-T^{2 / 3} \frac{a+\frac{u}{a}}{2}+T^{1 / 3} \frac{\left(\frac{u}{a}+a\right)^{2}}{4}\right\rfloor}(T) \geq T^{2 / 3} \frac{u}{a}-\frac{T^{1 / 3}}{2^{1 / 3}} s\right) \\
& =\lim _{\beta \rightarrow 0} \lim _{T \rightarrow \infty} \mathbb{P}\left(\tilde{x}_{\left\lfloor T \frac{(1-\beta)^{2}}{4}+\xi T^{1 / 3}\right\rfloor}(T) \geq T^{1 / 3} \frac{u}{\beta}-\frac{T^{1 / 3}}{2^{1 / 3}} s\right) \\
& =F_{\mathrm{GUE}}(s) F_{\mathrm{GUE}}\left(s-u 2^{4 / 3}\right) .
\end{aligned}
$$

Note that if we again formally set $a=\beta T^{1 / 3}$, and take $u \in \mathbb{R}, \xi=\frac{u}{2} \frac{\beta-1}{\beta}$, then we have

$$
\frac{T}{4}-T^{2 / 3} \frac{a+\frac{u}{a}}{2}+T^{1 / 3} \frac{\left(\frac{u}{a}+a\right)^{2}}{4}=T \frac{(1-\beta)^{2}}{4}+\xi T^{1 / 3}+\mathcal{O}\left(T^{-1 / 3}\right),
$$

which motivates the choice of the particle number in (1.4).

Finally, the identity (1.6) is a simple consequence of the known convergence (1.13). See also after Theorem 2.1 for a further discussion of this result.

1.1. TASEP and Last Passage Percolation. Here we introduce the notation for the two models considered in this paper, namely TASEP and Last Passage Percolation (LPP). We consider TASEP with particles labelled from right to left, i.e., when $x_{n}(T)$ denotes the position of particle number $n \in \mathbb{Z}$ at time $T$ we have

$$
\cdots<x_{2}(0)<x_{1}(0)<x_{0}(0)<x_{-1}(0)<x_{-2}(0) \cdots,
$$

note this order is preserved in time. TASEP is in one-to-one correspondence with last passage percolation, which we define next. Fix $(m, n) \in \mathbb{Z}^{2}$ (the end point) and $\mathcal{L} \subseteq \mathbb{Z}^{2}$ (the starting set). Let $\left\{\omega_{i, j}\right\}_{(i, j) \in \mathbb{Z}^{2}}$ be nonnegative random variables, seen as weights at the point $(i, j)$. An up-right path $\pi=(\pi(0), \ldots, \pi(k))$ from $\mathcal{L}$ to $(m, n)$ is a sequence of points with $\pi(0) \in \mathcal{L}, \pi(k)=(m, n), \pi(i)-\pi(i-1) \in\{(0,1),(1,0)\}$. Then the LPP time from $\mathcal{L}$ to $(m, n)$ is defined as

$$
L_{\mathcal{L} \rightarrow(m, n)}=\max _{\pi: \mathcal{L} \rightarrow(m, n)} \sum_{(i, j) \in \pi} \omega_{i, j}
$$

where the maximum in (1.9) is taken over all up-right paths from $\mathcal{L}$ to $(m, n)$. We will only consider $\left\{\omega_{i, j}\right\}_{(i, j) \in \mathbb{Z}^{2}}$ such that there is a.s. a unique path $\pi$ where the maximum (1.9) is attained, and we denote this path by $\pi^{\max }$. When there are no or infinitely many paths from $\mathcal{L}$ to $(m, n)$, we set, say, $L_{\mathcal{L} \rightarrow(m, n)}=\infty$, also (1.9) straightforwardly generalizes to several end points.

Given an initial data $\left\{x_{n}(0)\right\}_{n \in I}, I \subset \mathbb{Z}$, of TASEP we set $\mathcal{L}=\left\{\left(x_{n}(0)+\right.\right.$ $n, n), n \in I\}$. Assuming all particles have an exponential clock with parameter 1 we take $\left\{\omega_{i, j}\right\}_{(i, j) \in \mathbb{Z}^{2}}$ independent, and $\omega_{i, j} \sim \exp (1)$ if $(i, j) \notin \mathcal{L}$ and $\omega_{i, j}=0$ for $(i, j) \in \mathcal{L}$. With this choice, the link between TASEP and LPP is given by

$$
\mathbb{P}\left(x_{n}(T) \geq m-n\right)=\mathbb{P}\left(L_{\mathcal{L} \rightarrow(m, n)} \leq T\right) .
$$


1.2. Related Works. Here we rehash some of the known results that we will use in this paper, and describe the method of proof from Ferrari and Nejjar (2015a), which is relevant to this paper.

A result we shall use repeatedly in this paper concerns the transversal fluctuations of the maximizer $\pi^{\max }$ : when $\omega_{i, j}$ are i.i.d. and $\omega_{i, j} \sim \exp (1)$, there are bounds on the probability that the maximizer $\pi^{\max }$ from $(0,0)$ to a point $(\tau T, T)$ deviates more than $k T^{2 / 3}$ from the straight line $\{(\kappa \tau T, \kappa T), 0 \leq \kappa \leq 1\}$. This is a result from Basu et al. (2016) and cited here as Theorem 3.2. Furthermore, for line-to-point problems, we have detailed control over the distribution of the (random) starting point of $\pi^{\max }$, see Pimentel (2017), Lemma 1.1, Lemma 1.2 and also (4.18) in Ferrari and Occelli (2018).

The fluctuation behavior at shocks, rarefaction fans and flat (constant density) TASEP have been obtained in detail. We collect here the relevant results.

i) Rarefaction fan: By Theorem 1.6 of Johansson (2000b), for the initial data (1.2) for $a=0$ we have that, for $u \in \mathbb{R}$

$$
\lim _{T \rightarrow \infty} \mathbb{P}\left(\frac{x_{\left\lfloor T / 4+u 2^{-2 / 3} T^{2 / 3}\right\rfloor}(T)+u 2^{1 / 3} T^{2 / 3}-u^{2} T^{1 / 3} 2^{-1 / 3}}{-T^{1 / 3} 2^{-1 / 3}} \leq s\right)=F_{\mathrm{GUE}}(s) .
$$

ii) Flat TASEP: For flat TASEP, we have the following result, which is a reformulation of Theorem 2.8 in Ferrari and Occelli (2018). For $\varrho \in(0,1)$, $x_{n}^{\varrho}(0)=-\lfloor n / \varrho\rfloor$ and $u \in \mathbb{R}$, we have that

$$
\lim _{T \rightarrow \infty} \mathbb{P}\left(x_{\left\lfloor\varrho(1-\varrho) T+u T^{2 / 3}\right\rfloor}^{\varrho}(T) \geq-\frac{u}{\varrho} T^{2 / 3}-\frac{(1-\varrho)^{2 / 3}}{\varrho^{1 / 3}} T^{1 / 3} s\right)=F_{\mathrm{GOE}}\left(2^{2 / 3} s\right) .
$$

iii) Shocks: In the shock case (1.3), we have (see Ferrari and Nejjar, 2015a, Corollary 2.7) that

$$
\begin{aligned}
\lim _{T \rightarrow \infty} \mathbb{P}\left(\tilde{x}_{\left\lfloor\frac{(1-\beta)^{2}}{4} T+\xi T^{1 / 3}\right\rfloor}(T) \geq-s T^{1 / 3}\right)= & F_{\mathrm{GUE}}\left(\frac{s-\xi / \rho_{1}}{\sigma_{1}}\right) \\
& \times F_{\mathrm{GUE}}\left(\frac{s-\xi / \rho_{2}}{\sigma_{2}}\right)
\end{aligned}
$$

$$
\text { for } \xi \in \mathbb{R}, \rho_{1}=\frac{1-\beta}{2}, \rho_{2}=\frac{1+\beta}{2}, \sigma_{1}=\frac{(1+\beta)^{2 / 3}}{2^{1 / 3}(1-\beta)^{1 / 3}} \text {, and } \sigma_{2}=\frac{(1-\beta)^{2 / 3}}{2^{1 / 3}(1+\beta)^{1 / 3}} \text {. }
$$

In fact, in Ferrari and Nejjar (2015a), a general Theorem is presented which is then applied to other shock initial data as well. This general Theorem is formulated in terms of LPP. By the link (1.10) the distribution of the particle position at the shock (e.g. $\tilde{x}_{\left\lfloor\frac{(1-\beta)^{2}}{4} T+\xi T^{1 / 3}\right\rfloor}(T)$ from (1.13)) is equivalent to studying $\max \left\{L_{\mathcal{L}^{-} \rightarrow E}, L_{\mathcal{L}^{+} \rightarrow E}\right\}$ where $E \in \mathbb{Z}^{2}$ is chosen to be at the shock and $\mathcal{L}^{+}$(resp. $\mathcal{L}^{-}$) lie in the upper left (resp. lower right) quadrant. The main observation of Ferrari and Nejjar (2015a) is that $L_{\mathcal{L}^{-} \rightarrow E}, L_{\mathcal{L}^{+} \rightarrow E}$ decouple as $T \rightarrow \infty$.

This decoupling is based on three facts:

i) The maximizers $\pi_{\mathcal{L}^{+} \rightarrow E}^{\max }, \pi_{\mathcal{L}^{-} \rightarrow E}^{\max }$ start at points with distance $\mathcal{O}(T)$ with probability 1 as $T \rightarrow \infty$.

ii) The transversal fluctuations of $\pi_{\mathcal{L}^{+} \rightarrow E}^{\max }, \pi_{\mathcal{L}^{-} \rightarrow E}^{\max }$ are $\mathcal{O}\left(T^{2 / 3}\right)$. [In fact, in Ferrari and Nejjar (2015a) it suffices to know they are $o\left(T^{\chi}\right)$ for some $\chi<1$. 
iii) The slow decorrelation phenomenon Corwin et al. (2012): Consider a point $E^{+}$on the characteristic line joining $\mathcal{L}^{+}$and $E$ (the characteristic line is the deterministic line which $\pi_{\mathcal{L}^{+} \rightarrow E}^{\max }$, on the $\mathcal{O}(T)$ scale, follows). If $\left\|E^{+}-E\right\|=\mathcal{O}\left(T^{\nu}\right), \nu<1$, then $T^{-1 / 3}\left(L_{\mathcal{L}^{+} \rightarrow E+}+\mu T^{\nu}-L_{\mathcal{L}^{+} \rightarrow E}\right)$ converges to zero in probability when choosing the right value for $\mu$.

Point iii) allows to replace $L_{\mathcal{L}^{+} \rightarrow E}$ by $L_{\mathcal{L}^{+} \rightarrow E^{+}}$. If we take $\nu>2 / 3$, then by points i), ii), $\pi_{\mathcal{L}^{+} \rightarrow E^{+}}^{\max }, \pi_{\mathcal{L}^{-} \rightarrow E}^{\max }$ stay in (deterministic) disjoint sets with very high probability, i.e. $L_{\mathcal{L}^{-} \rightarrow E}, L_{\mathcal{L}^{+} \rightarrow E^{+}}$are asymptotically independent, leading to the result.

Furthermore, in Ferrari and Nejjar (2015b), we had studied the critical scaling for flat TASEP, where the shock is created by the presence of different speeds in the model, and numerically obtained the transition to the product structure of Ferrari and Nejjar (2015a). Finally, after this work was posted on arxiv, the transition to shock fluctuations for flat TASEP was obtained in the recent work Quastel and Rahman (2018), by completely different methods than ours, namely new exact determinantal formulas for TASEP, and without using the LPP picture.

1.3. Contributions of this paper. This paper is the first to study the transition of fluctuations when the density is smooth to the fluctuations when there is a shock. Corollary 1.1, and all other results in this paper, are obtained by working in the last passage percolation (LPP) picture. In terms of LPP, studying the transition to shock fluctuations means to study the maximum of two last passage times which remain correlated for all $T>0$, but which, as we show, decouple in a double limit $\lim _{a \rightarrow \infty} \lim _{T \rightarrow \infty}$, where $a$ is an extra parameter in the TASEP/LPP model.

As a concrete model, corresponding to the initial data (1.2), we consider in Theorem 2.4 for $a \geq 0$ the starting sets $\mathcal{L}^{+}=\left(-\left\lfloor a T^{2 / 3}\right\rfloor, 0\right), \mathcal{L}^{-}=\left(0,-\left\lfloor a T^{2 / 3}\right\rfloor\right)$ and an end point $E=\left(\left\lfloor T+\frac{u}{a} T^{2 / 3}\right\rfloor,\lfloor T\rfloor\right)$. For $a>0$, we are in a critical scaling. A lower bound for $\mathbb{P}\left(\max \left\{L_{\mathcal{L}^{+} \rightarrow E}, L_{\mathcal{L}^{-} \rightarrow E}\right\} \leq s\right)$ is provided by the FKG inequality, so the main work is to find a suitable upper bound, which we do in Theorems 2.1 and 2.4. These bounds in particular imply that one recovers, in the double limit $\lim _{a \rightarrow \infty} \lim _{T \rightarrow \infty}$, the product structure of Ferrari and Nejjar (2015a) as in Corollary 1.1.

One can deduce from Theorem 2.4 a statement about the decoupling of the two-point function of the Airy f $_{2}$ process, see Corollary 2.5. While more precise statements than ours are available (see Shinault and Tracy, 2011, Widom, 2004, Adler and van Moerbeke, 2005), our proof is new and probabilistic as we make use of the convergence in LPP; which gives some intuition as to why the decoupling happens.

We also consider shocks which, unlike in (1.13), are not between two regions of decreasing density, but two regions of (different) constant densities $\varrho_{1}>\varrho_{2}$. In this case, however, the fluctuations of the macroscopic shock, i.e. the analogue of (1.13), has not been obtained ${ }^{1}$, but see Ferrari et al. $(2018+)$, Section 2 for computations in this direction.

We prove the fluctuations for such a macroscopic shock in Theorem 2.2, which gives a product of two $F_{\mathrm{GOE}}$ distributions. The analogue of Corollary 1.1, i.e. the transition of the fluctuations of TASEP with constant density to the fluctuations

\footnotetext{
${ }^{1}$ In Corollary 2.5 of Ferrari and Nejjar (2015a), a shock between regions of constant density was considered, which is however created by slow particles.
} 
of Theorem 2.2, is stated in (2.10), the proof is however only sketched at the end of Section 4.

Theorem 2.4 can be seen as an instance of a general Theorem about the decoupling of last passage times under some assumptions, see Theorem 2.8. Theorem 2.8 is much simpler than the general Theorem 2.1 of Ferrari and Nejjar (2015a), and at the same time, gives a stronger result, as it provides some upper and lower bounds. Furthermore, Theorem 2.8 gives the framework to show the decoupling of the Airy ${ }_{1}$, Airy $_{2 \rightarrow 1}$ processes (see Borodin et al., 2008, Borodin and Ferrari, 2008 for definitions), which has not been done before, see Theorem 2.6. The decoupling of these processes corresponds to the decoupling of last passage times $L_{\mathcal{L} \rightarrow E_{1}}, L_{\mathcal{L} \rightarrow E_{2}}$ where $\mathcal{L}$ is now a (half-) line and the points $E_{1}, E_{2}$ have distance $a T^{2 / 3}$ from each other. Finally, as the simplest example of decoupling, we show in Theorem 2.7 the decoupling along the time-like direction in exponential LPP. Recently, exact formulas (Johansson, 2017, 2018) for the two time distribution have been found, and such a decoupling had been expected to occur, see Johansson (2017), Remark 2.3 .

1.3.1. Methods of proof. Let us briefly describe how our methods differ from related work, in particular Ferrari and Nejjar (2015a) (see Section 1.2). First, and unlike in Ferrari and Nejjar (2015a), the maximizing paths now start at distance $\mathcal{O}\left(T^{2 / 3}\right)$ from each other, which is the scale of their transversal fluctuations. This requires us to use a refined control over transversal fluctuations of maximizers in LPP, using results from Basu et al. (2016), see Theorem 3.2. The second main ingredient to Theorem 2.4 is an extended slow decorrelation result. Namely, since we have two maximizers which start in points with distance $\mathcal{O}\left(T^{2 / 3}\right)$ and go to $E$, the maximizers will come together already at distance $\mathcal{O}(T)$ from $E$. Consequently, in order to obtain independent passage times, we wish to replace $L_{\mathcal{L}^{+} \rightarrow E}$ by $L_{\mathcal{L}^{+} \rightarrow E^{+}}$, with $E^{+}$on the straight (characteristic) line from $\mathcal{L}^{+}$to $E$ and at distance $\varepsilon T$ from $E$. If $\varepsilon$ is not too small, the probability that the maximizers of $L_{\mathcal{L}^{+} \rightarrow E^{+}}, L_{\mathcal{L}^{-} \rightarrow E}$ cross will vanish for $a, T$ large. In the usual slow decorrelation (see Theorem 2.1 in Corwin et al., 2012), used in Ferrari and Nejjar (2015a), $E^{+}$is at distance $T^{\nu}, \nu<1$, from $E$ such that the fluctuations from $E^{+}$to $E$ vanish under the $T^{1 / 3}$ scaling. In our situation, however, they do not vanish as $T \rightarrow \infty$. Nevertheless, they are only of order $\varepsilon^{1 / 3} T^{1 / 3}$; in particular, they vanish in the double $\operatorname{limit}_{\lim } \lim _{T \rightarrow \infty} \lim _{T \rightarrow \infty}$. We show that it is possible to choose $\varepsilon=\varepsilon(a)$ in such a way that $\varepsilon(a)$ goes to zero with $a$, but is large enough so that the maximizers stay in disjoint sets with high probability (see Section 3), leading to Theorem 2.4.

To show the decoupling of the Airy ${ }_{1}$, Airy $_{2 \rightarrow 1}$ processes, no slow decorrelation result is needed, but a control over transversal fluctuations and the (random) starting point of the maximizing path, the latter was obtained recently in Pimentel (2017) and Ferrari and Occelli (2018). Finally, for the macroscopic shock proven in Theorem 2.2, no extended slow decorrelation or refined control over transversal fluctuations and starting points is needed, but we do need the universality of the $F_{\mathrm{GOE}}$ distribution in flat TASEP, cited here in (1.12).

Outline. In Section 2 we state all the results obtained in this paper. They concern either point-to-point LPP problems, or line-to-point LPP problems. 
In Section 3 we prove all results that concern point-to-point problems, and the general Theorem 2.8. Specifically, Corollary 1.1, Theorem 2.1, Theorem 2.4, Corollary 2.5, Theorem 2.7 and Theorem 2.8 are proven in Section 3. In Section 4 we prove all results that concern line-to-point problems: These are Theorem 2.2 and Theorem 2.6. Finally, an outline of the proof for the transition to shock fluctuations for flat TASEP, stated in (2.10), is given.

Acknowledgements We thank Márton Balázs for discussing Balázs et al. (2006) with us, and Patrik Ferrari and Zhipeng Liu for useful discussions regarding this paper, as well as the anonymous referee for helpful comments.

Notation We denote for $x \in \mathbb{R}$ by $\lfloor x\rfloor$ the largest $z \in \mathbb{Z}$ with $z \leq x$, and $T, t$ are always large time parameters which go to infinity. $C, c$ denote constants whose exact values are immaterial and do not depend on the parameters present (mostly $a, t, k)$.

\section{Main Results}

2.1. Transition to Shock Fluctuations. The following Theorem provides the transition from the fluctuations of TASEP with step initial data to the fluctuations at the GUE - GUE shock of (1.13).

Theorem 2.1. Let $x_{n}(0)=-n$ for $-\left\lfloor a T^{2 / 3}\right\rfloor \leq n \leq 0$ and $x_{n}(0)=-n-\left\lfloor a T^{2 / 3}\right\rfloor$ for $n \geq 1$. Then there are constants $C, c>0$ such that for any $0<k<a, \delta>0$ and $\frac{k}{a}<\varepsilon(a)<1$ we may bound

$$
\begin{aligned}
& F_{\mathrm{GUE}}(s) F_{\mathrm{GUE}}\left(s-u 2^{4 / 3}\right) \\
& \leq \lim _{T \rightarrow \infty} \mathbb{P}\left(x_{\left\lfloor\frac{T}{4}-T^{2 / 3} \frac{a+\frac{u}{a}}{2}\right\rfloor}(T) \geq \frac{u}{a} T^{2 / 3}+T^{1 / 3} \frac{\left(\frac{u}{a}+a\right)^{2}}{2}-\frac{T^{1 / 3}}{2^{1 / 3}} s\right) \\
& \leq F_{\mathrm{GUE}}\left(\frac{s+\delta}{(1-\varepsilon(a))^{1 / 3}}\right) F_{\mathrm{GUE}}\left(s-u 2^{4 / 3}\right) \\
& +F_{\mathrm{GUE}}\left(-\delta \varepsilon(a)^{-1 / 3}\right)+C e^{-c k} .
\end{aligned}
$$

In particular, Theorem 2.1 directly implies the following:

$$
\begin{array}{r}
\lim _{a \rightarrow \infty} \lim _{T \rightarrow \infty} \mathbb{P}\left(x_{\left\lfloor\frac{T}{4}-T^{2 / 3} \frac{a+\frac{u}{a}}{2}\right\rfloor}(T) \geq \frac{u}{a} T^{2 / 3}+T^{1 / 3} \frac{\left(\frac{u}{a}+a\right)^{2}}{2}-\frac{T^{1 / 3}}{2^{1 / 3}} s\right) \\
=F_{\mathrm{GUE}}(s) F_{\mathrm{GUE}}\left(s-u 2^{4 / 3}\right) .
\end{array}
$$

By taking $u=a \tilde{u}$ in (2.1) such that $u / a=\tilde{u}$ and then setting $a=0$, one has the usual step initial data and the $T \rightarrow \infty$ limit in (2.1) gives the Airy $_{2}$ process $\mathcal{A}_{2}(\tilde{u})_{\tilde{u} \in \mathbb{R}}$. To recover the shock situation, one should transfer the $T^{1 / 3} \frac{\left(\frac{u}{a}+a\right)^{2}}{2}$ term in the particle number, i.e. consider

$$
\mathbb{P}\left(x_{\left\lfloor\frac{T}{4}-T^{2 / 3} \frac{a+\frac{u}{a}}{2}+T^{1 / 3} \frac{\left(\frac{u}{a}+a\right)^{2}}{4}\right\rfloor}(T) \geq \frac{u}{a} T^{2 / 3}-\frac{T^{1 / 3}}{2^{1 / 3}} s\right) .
$$

To create a macroscopic shock, set, for $\beta \in(0,1), a=\beta T^{1 / 3}, \xi=\frac{u}{2} \frac{\beta-1}{\beta}$, so that (2.2) becomes (recall $\tilde{x}_{n}$ from (1.3))

$$
\mathbb{P}\left(\tilde{x}_{\left\lfloor T \frac{(1-\beta)^{2}}{4}+\tilde{\xi} T^{1 / 3}\right\rfloor}(T) \geq T^{1 / 3} u / \beta-\frac{T^{1 / 3}}{2^{1 / 3}} s\right) .
$$


Now (1.13) implies

$$
\lim _{\beta \rightarrow 0} \lim _{T \rightarrow \infty} \mathbb{P}\left(\tilde{x}_{\left\lfloor T \frac{(1-\beta)^{2}}{4}+\tilde{\xi} T^{1 / 3}\right\rfloor}(T) \geq T^{1 / 3} u / \beta-\frac{T^{1 / 3}}{2^{1 / 3}} s\right)=F_{\mathrm{GUE}}(s) F_{\mathrm{GUE}}\left(s-u 2^{4 / 3}\right),
$$

and Corollary 1.1 follows from (2.1) and (2.4).

Next we state our result for the fluctuations of macroscopic shocks created by two regions of constant density, where the decoupling already happens in the $T \rightarrow \infty$ limit.

Theorem 2.2. Consider TASEP with initial data given by $1>\varrho_{1}>\varrho_{2}>0$ and

$$
x_{n}^{\varrho_{1}, \varrho_{2}}(0)= \begin{cases}-\left\lfloor n / \varrho_{1}\right\rfloor & \text { for } \quad n \leq 0 \\ -\left\lfloor n / \varrho_{2}\right\rfloor & \text { for } \quad n>0 .\end{cases}
$$

Then we have with $c_{i}=\left(1-\varrho_{i}\right)^{-2 / 3} \varrho_{i}^{1 / 3}, i=1,2$

$$
\begin{aligned}
\lim _{T \rightarrow \infty} \mathbb{P} & \left(x_{\left\lfloor\varrho_{1} \varrho_{2} T+\xi T^{1 / 3}\right\rfloor}^{\varrho_{1}}(T) \geq\left(1-\varrho_{1}-\varrho_{2}\right) T-s T^{1 / 3}\right) \\
& =F_{\mathrm{GOE}}\left(2^{2 / 3}\left(s-\xi / \varrho_{1}\right) c_{1}\right) F_{\mathrm{GOE}}\left(2^{2 / 3}\left(s-\xi / \varrho_{2}\right) c_{2}\right) .
\end{aligned}
$$

The following is imminent from Theorem 2.2.

Corollary 2.3. We have that with $\tilde{\xi}=\xi\left(\varrho_{1}-\varrho_{2}\right)^{-1}$

$$
\begin{gathered}
\lim _{\varrho_{1} \searrow \varrho_{2}} \lim _{T \rightarrow \infty} \mathbb{P}\left(x_{\left\lfloor\varrho_{1} \varrho_{2} T+\tilde{\xi} T^{1 / 3}\right\rfloor}^{\varrho_{1} \varrho_{2}}(T) \geq\left(1-\varrho_{1}-\varrho_{2}\right) T-\left(s / c_{1}+\tilde{\xi} / \varrho_{1}\right) T^{1 / 3}\right) \\
=F_{\mathrm{GOE}}\left(2^{2 / 3} s\right) F_{\mathrm{GOE}}\left(2^{2 / 3} s-2^{2 / 3} \xi\left(1-\varrho_{2}\right)^{-2 / 3} \varrho_{2}^{-5 / 3}\right) .
\end{gathered}
$$

Now, to be in a critical scaling, set $\varrho_{1}(a)=\varrho_{2}+a T^{-1 / 3}$ and recall $\tilde{\xi}=\xi\left(\varrho_{1}-\right.$ $\left.\varrho_{2}\right)^{-1}$. Then the analogue of Corollary 1.1 is

$$
\begin{aligned}
& \lim _{a \rightarrow+\infty} \lim _{T \rightarrow \infty} \mathbb{P}\left(x_{\left\lfloor\varrho_{1}(a) \varrho_{2} T+\xi T^{2 / 3} / a\right\rfloor}^{\varrho_{1}(a) \varrho_{2}}(T) \geq\left(1-\varrho_{1}(a)-\varrho_{2}\right) T-\xi T^{2 / 3} / a \varrho_{2}-s T^{1 / 3} / c_{2}\right) \\
& =\lim _{\varrho_{1} \searrow \varrho_{2} T \rightarrow \infty} \lim _{T \rightarrow \infty} \mathbb{P}\left(x_{\left\lfloor\varrho_{1} \varrho_{2} T+\tilde{\xi} T^{1 / 3}\right\rfloor}^{\varrho_{1} \varrho_{2}}(T) \geq\left(1-\varrho_{1}-\varrho_{2}\right) T-\left(s / c_{1}+\tilde{\xi} / \varrho_{1}\right) T^{1 / 3}\right) .
\end{aligned}
$$

We do not provide a full proof of (2.10), but see the end of Section 4 for a discussion and outline of proof of (2.10).

2.2. Decoupling of Last Passage Times. In the following, we give our results which correspond to several last passage times decoupling in a certain double limit. The first, Theorem 2.4, is the LPP counter part of Theorem 2.1. By using that various Airy processes arise as limit in LPP models, we show how decoupling of last passage times implies decoupling bounds for the Airy ${ }_{1}$, Airy $_{2}$ and Airy $_{2 \rightarrow 1}$ processes. The decoupling of the Airy ${ }_{2}$ process, reported in Corollary 2.5, is a corollary of Theorem 2.4, whereas the decoupling of the Airy ${ }_{2 \rightarrow 1}$, Airy ${ }_{1}$ processes require new proofs. Finally, all these decouplings of last passage times fall in the framework of a simple more general statement about decoupling of last passage times, see Theorem 2.8. This improves Theorem 2.1 of Ferrari and Nejjar (2015b).

Theorem 2.4. Set $\mathcal{L}^{+}=\left(-\left\lfloor a t^{2 / 3}\right\rfloor, 0\right), \mathcal{L}^{-}=\left(0,-\left\lfloor a t^{2 / 3}\right\rfloor\right), \mathcal{L}=\mathcal{L}^{+} \cup \mathcal{L}^{-}$and define

$$
\mu^{a} t=4 t+2 t^{2 / 3}(a+u / a)-\left(a+\frac{u}{a}\right)^{2} t^{1 / 3} / 4 .
$$


There are constants $C, c>0$ such that for $a>k>0$, any $\delta>0$ and $k / a<\varepsilon(a)<1$ we may bound

$$
\begin{aligned}
F_{\mathrm{GUE}}(s) F_{\mathrm{GUE}}\left(s-\frac{u}{2^{4 / 3}}\right) & \leq \lim _{t \rightarrow \infty} \mathbb{P}\left(\frac{L_{\mathcal{L} \rightarrow\left(\left\lfloor t+\frac{u}{a} t^{2 / 3}\right\rfloor,\lfloor t\rfloor\right)}-\mu^{a} t}{2^{4 / 3} t^{1 / 3}} \leq s\right) \\
& \leq F_{\mathrm{GUE}}\left(\frac{s+\delta}{(1-\varepsilon(a))^{1 / 3}}\right) F_{\mathrm{GUE}}\left(s-\frac{u}{2^{4 / 3}}\right) \\
& +F_{\mathrm{GUE}}\left(-\delta \varepsilon(a)^{-1 / 3}\right)+C e^{-c k} .
\end{aligned}
$$

2.3. Decoupling of Airy Processes. Theorem 2.4 gives some estimates on the decay of the two point function of the Airy 2 process $\mathcal{A}_{2}$. The two point function $\mathbb{P}\left(\mathcal{A}_{2}(0) \leq s_{1}, \mathcal{A}_{2}(a) \leq s_{2}\right)$ has already been studied in detail (see in particular (7) in Shinault and Tracy, 2011, and also the previous works Widom, 2004, Adler and van Moerbeke, 2005). In particular, it is known that $\mathbb{P}\left(\mathcal{A}_{2}(0) \leq s_{1}, \mathcal{A}_{2}(a) \leq s_{2}\right)=$ $F_{\mathrm{GUE}}\left(s_{1}\right) F_{\mathrm{GUE}}\left(s_{2}\right)+\mathcal{O}\left(a^{-2}\right)$ as $a \rightarrow \infty$. However, the works Shinault and Tracy (2011), Widom (2004), Adler and van Moerbeke (2005) are all based on Fredholm determinant (in Widom, 2004 and Shinault and Tracy, 2011) or PDE expression (in Adler and van Moerbeke, 2005) for the two point function, whereas we use that the Airy $_{2}$ process arises as limit in LPP.

Corollary 2.5. Let $a>k>0$. Then for any $\delta>0$ and $1>\varepsilon(a)>k / a$ we may bound

$$
\begin{aligned}
F_{\mathrm{GUE}}(s) F_{\mathrm{GUE}}(s-4 u) & \leq \mathbb{P}\left(\mathcal{A}_{2}\left(-a-\frac{u}{a}\right) \leq s, \mathcal{A}_{2}\left(a-\frac{u}{a}\right) \leq s-4 u\right) \\
& \leq F_{\mathrm{GUE}}\left(\frac{s+\delta}{(1-\varepsilon(a))^{1 / 3}}\right) F_{\mathrm{GUE}}(s-4 u) \\
& +F_{\mathrm{GUE}}\left(-\delta \varepsilon(a)^{-1 / 3}\right)+C e^{-c k}
\end{aligned}
$$

In Section 4, we also study the decay of the joint distribution of the Airy , Airy $_{2 \rightarrow 1}$ processes, which we denote by $\mathcal{A}_{1}, \mathcal{A}_{2 \rightarrow 1}$. This decoupling does not correspond to a transition to shock fluctuations, rather one has two maximizers which start and end in points with distance $a t^{2 / 3}$. The starting point is random, and controlling it is an extra ingredient required here, which was obtained recently in Ferrari and Occelli (2018) and Pimentel (2017). The result we obtain is as follows.

Theorem 2.6. There are $C, c, a_{0}>0$ such that for $a>a_{0}, b \in \mathbb{R}$ we have

$$
\begin{aligned}
F_{\mathrm{GOE}}\left(2 s_{1}\right) F_{\mathrm{GOE}}\left(2 s_{2}\right) & \leq \mathbb{P}\left(\mathcal{A}_{1}(0) \leq s_{1}, \mathcal{A}_{1}(a) \leq s_{2}\right) \\
& \leq F_{\mathrm{GOE}}\left(2 s_{1}\right) F_{\mathrm{GOE}}\left(2 s_{2}\right)+C e^{-c a}
\end{aligned}
$$

and that for the Airy $_{2 \rightarrow 1}$ process we may bound

$$
\begin{aligned}
& \mathbb{P}\left(\mathcal{A}_{2 \rightarrow 1}(b) \leq s_{1}\right) \mathbb{P}\left(\mathcal{A}_{2 \rightarrow 1}(|b|+a) \leq s_{2}\right) \\
& \leq \mathbb{P}\left(\mathcal{A}_{2 \rightarrow 1}(b) \leq s_{1}, \mathcal{A}_{2 \rightarrow 1}(|b|+a) \leq s_{2}\right) \\
& \leq \mathbb{P}\left(\mathcal{A}_{2 \rightarrow 1}(b) \leq s_{1}\right) \mathbb{P}\left(\mathcal{A}_{2 \rightarrow 1}(|b|+a) \leq s_{2}\right)+C e^{-c a} .
\end{aligned}
$$

2.4. Decoupling in the time-like direction. Finally, as the simplest example of this paper, we show the decoupling of last passage percolation times along the time-like 
direction. Denote for $x, y \in \mathbb{R}$ the points $P(x, y)=\left(\left\lfloor-y(x t)^{2 / 3}\right\rfloor, 0\right)$ and $\mu(x, y) t=$ $4 x t-2 y(x t)^{2 / 3}+\frac{y^{2}}{4}(x t)^{1 / 3}$ and denote

$$
L_{P(x, y) \rightarrow(\lfloor x t\rfloor,\lfloor x t\rfloor)}^{\mathrm{resc}}=\frac{L_{P(x, y) \rightarrow(\lfloor x t\rfloor,\lfloor x t\rfloor)}-\mu(x, y) t}{2^{4 / 3}(x t)^{1 / 3}}
$$

For e.g. points lying on a line with slope 1, the decoupling we consider corresponds to look for $\tau<a$ at

$$
\lim _{t \rightarrow \infty} \mathbb{P}\left(\left\{L_{0 \rightarrow(\lfloor\tau t\rfloor,\lfloor\tau t\rfloor)}^{\mathrm{resc}} \leq s\right\} \cap\left\{L_{0 \rightarrow(\lfloor a t\rfloor,\lfloor a t\rfloor)}^{\mathrm{resc}} \leq \zeta\right\}\right)
$$

and then let $a$ go to infinity. It is a priori not clear if (2.17) exists, hence we work with an arbitrary subsequential limit in (2.19). For the case of brownian and geometric percolation, Johansson proved in Johansson $(2017,2018)$ an explicit formula for (2.17), see also Baik and Liu (2018) for results in periodic TASEP, and Ferrari and Spohn (2016) for the decay of the covariance in the time-like direction. In Johansson (2017), the author expects (see Remark 2.3 in Johansson, 2017) that (the analogue of) (2.17) converges to $F_{\mathrm{GUE}}(s) F_{\mathrm{GUE}}(\zeta)$ as $a \rightarrow \infty$ and notes that this can be checked heuristically but that it appears rather subtle. Here we show that a soft probabilistic argument suffices to show this decoupling, which extends to the multipoint two time distribution in exponential LPP, and even to provide some (non-optimal) bounds on the speed of decoupling. Note that the following Theorem implies in particular that

$$
\begin{aligned}
& \lim _{a \rightarrow \infty} \lim _{t_{j} \rightarrow \infty} \mathbb{P}\left(\bigcap_{i=1}^{l}\left\{L_{P\left(\tau, r_{i}\right) \rightarrow\left(\left\lfloor\tau t_{j}\right\rfloor,\left\lfloor\tau t_{j}\right\rfloor\right)}^{\mathrm{resc}} \leq s_{i}\right\} \cap \bigcap_{i=1}^{k}\left\{L_{P\left(a, u_{i}\right) \rightarrow\left(\left\lfloor a t_{j}\right\rfloor,\left\lfloor a t_{j}\right\rfloor\right)}^{\mathrm{resc}} \leq \zeta_{i}\right\}\right) \\
& =\mathbb{P}\left(\bigcap_{i=1}^{l} \mathcal{A}_{2}\left(r_{i}\right) \leq s_{i}\right) \mathbb{P}\left(\bigcap_{i=1}^{k} \mathcal{A}_{2}\left(u_{i}\right) \leq \zeta_{i}\right) \text {. }
\end{aligned}
$$

Theorem 2.7. Let $a>\tau>0$, and let the $\left\{\omega_{i, j}, i, j \in \mathbb{Z}\right\}$ be i.i.d. $\exp (1)$ distributed. Let $r_{1}<\cdots<r_{l}$ and $u_{1}<\cdots<u_{k}$. Denote by $\lim _{t_{j} \rightarrow \infty}$ an arbitrary subsequential limit. Then for any $\delta>0$

$$
\begin{aligned}
& \mathbb{P}\left(\bigcap_{i=1}^{l} \mathcal{A}_{2}\left(r_{i}\right) \leq s_{i}\right) \mathbb{P}\left(\bigcap_{i=1}^{k} \mathcal{A}_{2}\left(u_{i}\right) \leq \zeta_{i}\right) \\
& \leq \lim _{t_{j} \rightarrow \infty} \mathbb{P}\left(\bigcap_{i=1}^{l}\left\{L_{P\left(\tau, r_{i}\right) \rightarrow\left(\left\lfloor\tau t_{j}\right\rfloor,\left\lfloor\tau t_{j}\right\rfloor\right)}^{\mathrm{resc}} \leq s_{i}\right\} \cap \bigcap_{i=1}^{k}\left\{L_{P\left(a, u_{i}\right) \rightarrow\left(\left\lfloor a t_{j}\right\rfloor,\left\lfloor a t_{j}\right\rfloor\right)}^{\mathrm{resc}} \leq \zeta_{i}\right\}\right) \\
& \leq \mathbb{P}\left(\bigcap_{i=1}^{l} \mathcal{A}_{2}\left(r_{i}\right) \leq s_{i}\right) \mathbb{P}\left(\bigcap_{i=1}^{k} \mathcal{A}_{2}\left(u_{i}(1-\tau / a)^{1 / 3}\right) \leq\left(\zeta_{i}+\delta\right) \frac{a^{1 / 3}}{(a-\tau)^{1 / 3}}\right) \\
& +k F_{\mathrm{GUE}}\left(-\delta a^{1 / 3} \tau^{-1 / 3}\right) \text {. }
\end{aligned}
$$

2.5. General Theorem. The preceding results can all be phrased in a simple Theorem about a general LPP model, which improves the general framework given in Theorem 2.1 of Ferrari and Nejjar (2015a). Let $\mathcal{L}^{+}, \mathcal{L}^{-} \subseteq \mathbb{Z}^{2}$ and let $\left\{\omega_{i, j}, i, j \in \mathbb{Z}\right\}$ be independent exponentially distributed weights. We make three assumptions on our model. 
Assumption 1. Let $t, a>0$ and assume there are $E_{1}=E_{1}(t, a), E_{2}=E_{2}(t, a) \in \mathbb{Z}^{2}$ and $\mu_{1}^{a}, \mu_{2}^{a}>0$ such that

$$
\begin{aligned}
& \lim _{t \rightarrow \infty} \mathbb{P}\left(\frac{L_{\mathcal{L}^{+} \rightarrow E_{1}}-\mu_{1}^{a} t}{t^{1 / 3}} \leq s\right)=G_{1}^{a}(s) \\
& \lim _{t \rightarrow \infty} \mathbb{P}\left(\frac{L_{\mathcal{L}^{-} \rightarrow E_{2}}-\mu_{2}^{a} t}{t^{1 / 3}} \leq s\right)=G_{2}^{a}(s),
\end{aligned}
$$

where $G_{1}^{a}(s), G_{2}^{a}(s)$ are some distribution functions.

In Theorem 2.4, $G_{1}^{a}, G_{2}^{a}$ will be (shifted) $F_{\mathrm{GUE}}$ distributions, in Theorem 2.6, they will be $F_{\mathrm{GOE}}$ distributions.

Assumption 2. Assume there is a point $E^{+}=E_{1}-\left(\kappa \varepsilon(a) t+d t^{2 / 3}, \varepsilon(a) t\right)$ with $\kappa, \varepsilon(a) \geq 0, d \in \mathbb{R}$ such that for a $\mu^{\varepsilon(a)} \geq 0$ we have

$$
\begin{aligned}
& \lim _{t \rightarrow \infty} \mathbb{P}\left(\frac{L_{E^{+} \rightarrow E_{1}}-\mu^{\varepsilon(a)} t}{t^{1 / 3}} \leq s\right)=G_{0}^{a}(s) \\
& \lim _{t \rightarrow \infty} \mathbb{P}\left(\frac{L_{\mathcal{L}^{+} \rightarrow E^{+}}+\mu^{\varepsilon(a)} t-\mu_{1}^{a} t}{t^{1 / 3}} \leq s\right)=G_{1}^{a}\left(c_{\varepsilon(a)} s\right)
\end{aligned}
$$

where $G_{0}^{a}$ is a distribution function, $c_{\varepsilon(a)}$ is a constant and $G_{1}^{a}$ is from Assumption 1.

In the context of Theorem 2.4, we will take $\varepsilon(a)>0, \lim _{a \rightarrow \infty} \varepsilon(a)=0$. Then, with $E$ as in Theorem 2.4, $\frac{L_{E^{+} \rightarrow E}-\mu^{\varepsilon(a)} t}{t^{1 / 3}}$ will vanish in the double limit $\lim _{a \rightarrow \infty} \lim _{t \rightarrow \infty}$.

Assumption 3. Assume there are independent random variables $\tilde{L}_{\mathcal{L}^{+} \rightarrow E^{+}}$, $\tilde{L}_{\mathcal{L}^{-} \rightarrow E_{2}}$ such that for some $\tilde{\psi} \geq 0$

$$
\limsup _{t \rightarrow \infty} \mathbb{P}\left(\left\{\tilde{L}_{\mathcal{L}^{+} \rightarrow E^{+}} \neq L_{\mathcal{L}^{+} \rightarrow E^{+}}\right\} \cup\left\{\tilde{L}_{\mathcal{L}^{-} \rightarrow E_{2}} \neq L_{\mathcal{L}^{-} \rightarrow E_{2}}\right\}\right) \leq \tilde{\psi}
$$

In Theorem 2.4, $\tilde{L}_{\mathcal{L}^{+} \rightarrow E^{+}}, \tilde{L}_{\mathcal{L}^{-} \rightarrow E}$ will be last passage times with restricted transversal fluctuations, in Theorem 2.6 they will additionally have restricted starting points.

We denote by

$$
L_{\mathcal{L}^{+} \rightarrow E_{1}}^{\mathrm{resc}}=\frac{L_{\mathcal{L}^{+} \rightarrow E_{1}}-\mu_{1}^{a} t}{t^{1 / 3}}
$$

and similarly denote by $L_{\mathcal{L}^{+} \rightarrow E_{1}}^{\text {resc }}, L_{E^{+} \rightarrow E_{1}}^{\text {resc }}$ the LPP times rescaled as in Assumptions 1,2 .

Theorem 2.8. Under Assumptions 1, 2, 3 we have for any $\delta \geq 0$

$$
\begin{aligned}
G_{1}^{a}\left(s_{1}\right) G_{2}^{a}\left(s_{2}\right) & \leq \lim _{t_{k} \rightarrow \infty} \mathbb{P}\left(L_{\mathcal{L}^{+} \rightarrow E_{1}}^{\mathrm{resc}} \leq s_{1}, L_{\mathcal{L}^{-} \rightarrow E_{2}}^{\mathrm{resc}} \leq s_{2}\right) \\
& \leq G_{1}^{a}\left(\left(s_{1}+\delta\right) c_{\varepsilon(a)}\right) G_{2}^{a}\left(s_{2}\right)+G_{0}^{a}(-\delta)+3 \tilde{\psi},
\end{aligned}
$$

where $\lim _{t_{k} \rightarrow \infty}$ is any subsequential limit.

Clearly, a version of Theorem 2.8 without taking the $t_{k} \rightarrow \infty$ limit also holds. This could be used to refine the results of Ferrari and Nejjar (2015a) by obtaining upper and lower bounds for finite $t$ in Theorem 2.1 in Ferrari and Nejjar (2015a) and its applications, instead of showing only the convergence to a product as $t \rightarrow \infty$. 


\section{Proof for point-to-point problems and Theorem 2.8}

In this section, we prove the results which involve point(s)-to-point LPP problems, as well as the general Theorem 2.8. Corollary 1.1, Theorems 2.1, 2.4 and Corollary 2.5 are proved in Section 3.1, Theorem 2.7 and Theorem 2.8 are proved in Section 3.2.

3.1. Proof of Theorems 2.1, 2.4 and Corollaries 1.1, 2.5. The proof of Corollary 1.1 is immediate.

Proof of Corollary 1.1: It follows from (2.1) and (2.4).

Let us recall the following result for point-to-point LPP.

Proposition 3.1 (Theorem 1.6 of Johansson, 2000a, Theorem 2 of Borodin and Péché, 2008). Let $0<\eta<\infty, \eta=\eta_{0}+c \ell^{-1 / 3}$. Then,

$$
\lim _{\ell \rightarrow \infty} \mathbb{P}\left(L_{0 \rightarrow(\lfloor\eta \ell\rfloor,\lfloor\ell\rfloor)} \leq \mu_{\mathrm{pp}} \ell+s \sigma_{\eta} \ell^{1 / 3}\right)=F_{\mathrm{GUE}}(s)
$$

where $\mu_{\mathrm{pp}}=(1+\sqrt{\eta})^{2}$, and $\sigma_{\eta}=\eta^{-1 / 6}(1+\sqrt{\eta})^{4 / 3}$. In particular, with $\mathcal{L}^{+}, \mathcal{L}^{-}, \mu^{a} t$ as in Theorem 2.4, we have

$$
\begin{aligned}
& \lim _{t \rightarrow \infty} \mathbb{P}\left(\frac{L_{\mathcal{L}^{+} \rightarrow\left(t+u t^{2 / 3} / a, t\right)}-\mu^{a} t}{2^{4 / 3} t^{1 / 3}} \leq s\right)=F_{\mathrm{GUE}}(s) \\
& \lim _{t \rightarrow \infty} \mathbb{P}\left(\frac{L_{\mathcal{L}^{-} \rightarrow\left(t+u t^{2 / 3} / a, t\right)}-\mu^{a} t}{2^{4 / 3} t^{1 / 3}} \leq s\right)=F_{\mathrm{GUE}}\left(s-u / 2^{4 / 3}\right) .
\end{aligned}
$$

To proceed, we need bounds on the transversal fluctuations of maximizers in LPP. Let $(m, n) \in \mathbb{Z}_{>0}^{2}$. Denote for $l \leq n$

$$
Z_{l}(m, n)=Z_{l}=\max \left\{i:(i, l) \in \pi_{0 \rightarrow(m, n)}^{\max }\right\}
$$

where, with $A, B \in \mathbb{Z}_{>0}^{2}, \pi_{A \rightarrow B}^{\max }$ is the maximizing path from $A$ to $B$ in the LPP model with independent weights given by $\omega_{i, j} \sim \exp (1)$. Similarly, define $Y_{r}^{\mathrm{TOP}}(m, n)$ to be the top-most point of $\pi_{0 \rightarrow(m, n)}^{\max }$ on the vertical line $i=r$. The following result was formulated for Poisson LPP (and for lines with bounded slope), but extends to the exponential model straightforwardly, see Section 13 in Basu et al. (2016).

Theorem 3.2 (Corollary 11.7 in Basu et al., 2016). Let $d=d(t)=d_{0}+\mathcal{O}\left(t^{-1 / 3}\right)$, $d_{0} \in \mathbb{R}$. Set $m=\left\lfloor\eta_{0} t+d t^{2 / 3}\right\rfloor, n=\lfloor t\rfloor$. There are constants $C, c,>0$ such that for all $k>0$

$$
\begin{aligned}
& \limsup _{t \rightarrow \infty} \mathbb{P}\left(\max _{\kappa \in[0,1]}\left\{Z_{\lfloor\kappa t\rfloor}(m, n)-\kappa\left(\eta_{0} t+d t^{2 / 3}\right)\right\} \geq k t^{2 / 3}\right) \leq C e^{-c k} \\
& \limsup _{t \rightarrow \infty} \mathbb{P}\left(\max _{\kappa \in[0,1]}\left\{Y_{\left\lfloor\kappa\left(\eta_{0} t+d t^{2 / 3}\right)\right\rfloor}^{\mathrm{TOP}}(m, n)-\kappa t\right\} \geq k t^{2 / 3}\right) \leq C e^{-c k} .
\end{aligned}
$$

Next we choose the point $E^{+}$from Assumption 2. From Proposition 3.1 one can easily compute that $E^{+}$should lie on the line segment from $\mathcal{L}^{+}$to $E=(\lfloor t+$ $\left.\left.\frac{u}{a} t^{2 / 3}\right\rfloor,\lfloor t\rfloor\right)$, so it remains to choose $\varepsilon(a)$. To motivate this choice, note that by Theorem 3.2 we can control the probability that $\pi_{\mathcal{L}^{+} \rightarrow E}^{\max }, \pi_{\mathcal{L}^{-} \rightarrow E}^{\max }$ have transversal fluctuations of order $k t^{2 / 3}$. In particular, we have a good upper bound for the probability that $\pi_{\mathcal{L}^{+} \rightarrow E}^{\max }$ contains no point of the straight line $R_{+}$which joins (in $\mathbb{Z}^{2}$, see (3.12)) the points $\left(\left\lfloor-a t^{2 / 3}+k t^{2 / 3}\right\rfloor, 0\right)$ and $E+\left(\left\lfloor k t^{2 / 3}\right\rfloor, 0\right)$ and for the probability 


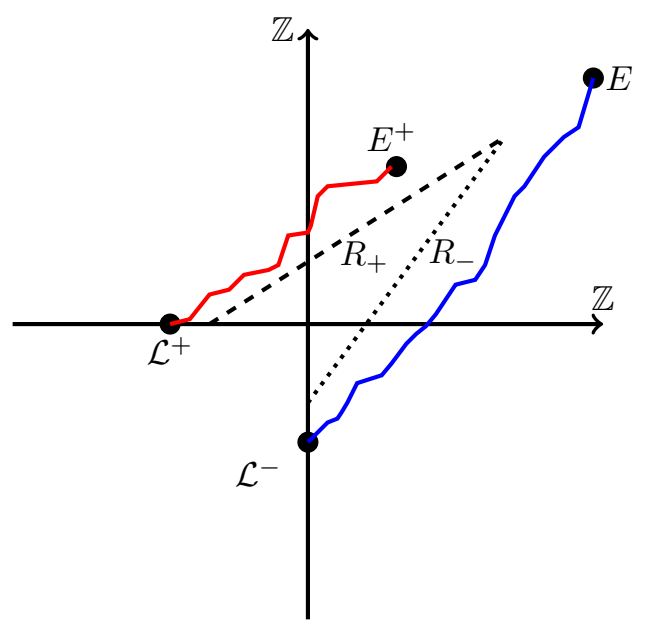

Figure 3.2. We choose $k=k(a)$ such that $\lim _{a \rightarrow \infty} k(a)=$ $\infty, \lim _{a \rightarrow \infty} \frac{k(a)}{a}=0, \varepsilon(a)>\frac{k}{a}$. Then, the maximizing path (blue) from $\mathcal{L}^{-}=\left(0,-\left\lfloor a t^{2 / 3}\right\rfloor\right)$ to $E=\left(\left\lfloor t+\frac{u}{a} t^{2 / 3}\right\rfloor,\lfloor t\rfloor\right)$ crosses the line segment $R_{-}=R_{-}(k)$ (dotted) with vanishing probability as $\lim _{a \rightarrow \infty} \lim _{t \rightarrow \infty}$. The point $E^{+}$is at distance $\varepsilon(a) t$ from $E$ on the line connecting $E$ with $\mathcal{L}^{+}=\left(-\left\lfloor a t^{2 / 3}\right\rfloor, 0\right)$ (see (3.7)). The maximizer from $\mathcal{L}^{+}$to $E^{+}$crosses $R_{+}=R_{+}(k)$ (dashed) with vanishing probability. So the two maximizers do not cross asymptotically, leading to the decoupling.

that $\pi_{\mathcal{L}^{-} \rightarrow E}^{\max }$ contains no point of the straight line $R_{-}$joining $\left(0,\left\lfloor-a t^{2 / 3}+k t^{2 / 3}\right\rfloor\right)$ and $E+\left(0,\left\lfloor k t^{2 / 3}\right\rfloor\right)$. Now an elementary calculation reveals that $R_{-}$and $R_{+}$cross in a point

$$
\left(\left\lfloor t\left(1-\frac{k}{a}\right)+\mathcal{O}\left(t^{2 / 3}\right)\right\rfloor,\left\lfloor t\left(1-\frac{k}{a}\right)+\mathcal{O}\left(t^{2 / 3}\right)\right\rfloor\right),
$$

see Figure 3.2.

In view of Assumption 3, we thus should choose $\varepsilon(a)>\frac{k}{a}$, though to satisfy Assumption 2, this is not necessary, as the following result shows.

Proposition 3.3. Let $1>\varepsilon(a)>0$. Then Assumption 2 holds with

$$
\begin{aligned}
& E^{+}=\left(\left\lfloor t(1-\varepsilon(a))+t^{2 / 3}(u / a-\varepsilon(a)(u / a+a))\right\rfloor,\lfloor t(1-\varepsilon(a))\rfloor\right) \\
& \mu^{\varepsilon(a)} t=4 \varepsilon(a) t+2 \varepsilon(a)(u / a+a) t^{2 / 3}-\frac{\varepsilon(a)(a+u / a)^{2}}{4} t^{1 / 3} \\
& c_{\varepsilon(a)}=(1-\varepsilon(a))^{-1 / 3} . \\
& G_{0}^{a}(s)=F_{\mathrm{GUE}}\left(s \varepsilon(a)^{-1 / 3}\right) .
\end{aligned}
$$

Proof: We have $L_{\mathcal{L}^{+} \rightarrow E^{+}}={ }^{d} L_{0 \rightarrow\left(\left(\left\lfloor t(1-\varepsilon(a))+t^{2 / 3} r_{1}\right\rfloor,\lfloor t(1-\varepsilon(a))\rfloor\right)\right.}$ for $r_{1}=(u / a+$ a) $(1-\varepsilon(a))$. The $\mu_{\mathrm{pp}}$ of Proposition 3.1 for $L_{\mathcal{L}^{+} \rightarrow E^{+}}$is given by $\mu_{\mathrm{pp}} t=4 t(1-$ $\varepsilon(a))+2 t^{2 / 3} r_{1}-\frac{r_{1}^{2}}{4(1-\varepsilon(a))} t^{1 / 3}$ and the one, with $E=\left(\left\lfloor t+\frac{u}{a} t^{2 / 3}\right\rfloor,\lfloor t\rfloor\right)$, of $L_{E^{+} \rightarrow E}$ equals $\mu^{\varepsilon(a)} t=4 t \varepsilon(a)+2 t^{2 / 3} r_{2}-\frac{r_{2}^{2}}{4 \varepsilon(a)} t^{1 / 3}$, with $r_{2}=-r_{1}+u / a+a$ and since the 
two terms need to sum up to $\mu^{a}$ from Theorem 2.4 we obtain the condition

$$
\frac{r_{2}^{2}}{4 \varepsilon(a)}+\frac{r_{1}^{2}}{4(1-\varepsilon(a))}=\frac{(u / a+a)^{2}}{4},
$$

which is precisely solved by our $r_{1}$. Finally, $c_{\varepsilon(a)}$ and $G_{0}^{a}$ are immediately obtained from Proposition 3.1.

Let now $E^{+}$be as in (3.7) and denote $E^{+, k}=E^{+}+\left(k t^{2 / 3}, 0\right)$. Define $\mathfrak{R}_{+}(k)=$ $\overline{\left(\left\lfloor-a t^{2 / 3}+k t^{2 / 3}\right\rfloor, 0\right) E^{+, k}}$ as the line segment $\left(\right.$ in $\left.\mathbb{R}^{2}\right)$ from $\left(\left\lfloor-a t^{2 / 3}+k t^{2 / 3}\right\rfloor, 0\right)$ to $E^{+, k}$, and denote

$$
R_{+}(k)=\left\{x \in \mathbb{Z}^{2}:|x-y| \leq 2 \text { for a } y \in \mathfrak{R}_{+}(k)\right\}
$$

a discrete approximation. See Figure 3.2. Denote by $\Pi^{+, k}$ the set of up-right paths from $\mathcal{L}^{+}$to $E^{+}$which do not contain any point of $R_{+}(k)$. Set

$$
\tilde{L}_{\mathcal{L}^{+} \rightarrow E^{+}}=\tilde{L}_{\mathcal{L}^{+} \rightarrow E^{+}}(k)=\max _{\pi \in \Pi^{+, k}} \sum_{(i, j) \in \pi} \omega_{i, j} .
$$

Let now $E=\left(\left\lfloor t+\frac{u}{a} t^{2 / 3}\right\rfloor,\lfloor t\rfloor\right)$ and $E^{k}=E+\left(0, k t^{2 / 3}\right)$. Write $\mathfrak{R}_{-}(k)=$ $\left(0,\left\lfloor-a t^{2 / 3}+k t^{2 / 3}\right\rfloor\right) E^{k}$ for the line segment in $\mathbb{R}^{2}$ joining $\left(0,\left\lfloor-a t^{2 / 3}+k t^{2 / 3}\right\rfloor\right)$ and $E^{k}$ and set

$$
R_{-}(k)=\left\{x \in \mathbb{Z}^{2}:|x-y| \leq 2 \text { for a } y \in \mathfrak{R}_{-}(k)\right\} .
$$

Define $\Pi^{-, k}$ to be the set of up-right paths from $\mathcal{L}^{-}$to $E$ which do not contain any point of $R_{-}(k)$. We define

$$
\tilde{L}_{\mathcal{L}^{-} \rightarrow E}=\tilde{L}_{\mathcal{L}^{-} \rightarrow E}(k)=\max _{\pi \in \Pi^{-}, k} \sum_{i, j \in \pi} \omega_{i, j} .
$$

Proposition 3.4. Let $a>k>0$ and let $E^{+}$be given by (3.7) with $1>\varepsilon(a)>\frac{k}{a}$ and let $\tilde{L}_{\mathcal{L}^{-} \rightarrow E}(k), \tilde{L}_{\mathcal{L}^{+} \rightarrow E^{+}}(k)$ be given by (3.15), (3.13). Then there are constants $c, C>0$ such that Assumption 3 holds with $\psi=C e^{-c k}$.

Proof: Note that we have $L_{\mathcal{L}^{+} \rightarrow E^{+}}={ }^{d} L_{0 \rightarrow\left(\left(\left\lfloor t(1-\varepsilon(a))+t^{2 / 3} r_{1}\right\rfloor,\lfloor t(1-\varepsilon(a))\rfloor\right)\right.}$ for $r_{1}=$ $(u / a+a)(1-\varepsilon(a))$. Write $\left(\left\lfloor t(1-\varepsilon(a))+t^{2 / 3} r_{1}\right\rfloor,\lfloor t(1-\varepsilon(a))\rfloor\right)=\left(m_{+}, n_{+}\right)$. Thus by translation invariance and Theorem 3.2

$$
\begin{aligned}
& \limsup _{t \rightarrow \infty} \mathbb{P}\left(L_{\mathcal{L}^{+} \rightarrow E^{+}} \neq \tilde{L}_{\mathcal{L}^{+} \rightarrow E^{+}}(k)\right) \\
& \leq \limsup _{t \rightarrow \infty} \mathbb{P}\left(\max _{\kappa \in[0,1]}\left\{Z_{\left\lfloor\kappa n_{+}\right\rfloor}\left(m_{+}, n_{+}\right)-\kappa m_{+}\right\} \geq k t^{2 / 3}\right) \leq C e^{-c k} .
\end{aligned}
$$

Furthermore,

$$
L_{\mathcal{L}^{-} \rightarrow E}={ }^{d} L_{0 \rightarrow\left(\left\lfloor t+\frac{u}{a} t^{2 / 3}\right\rfloor,\lfloor t\rfloor+\left\lfloor a t^{2 / 3}\right\rfloor\right)} .
$$

Setting $T=\lfloor t\rfloor+\left\lfloor a t^{2 / 3}\right\rfloor$ we have $t+\frac{u}{a} t^{2 / 3}=T+\left(\frac{u}{a}-a\right) T^{2 / 3}+\mathcal{O}\left(T^{1 / 3}\right)=$ $T+c_{-}(T) T^{2 / 3}$ for a $c_{-}(T)=\frac{u}{a}-a+\mathcal{O}\left(T^{-1 / 3}\right)$ such that

$$
L_{\mathcal{L}^{-} \rightarrow E}={ }^{d} L_{0 \rightarrow\left(\left\lfloor T+c_{-}(T) T^{2 / 3}\right\rfloor,\lfloor T\rfloor\right)} .
$$


We thus get

$$
\begin{aligned}
& \limsup _{t \rightarrow \infty} \mathbb{P}\left(L_{\mathcal{L}^{-} \rightarrow E} \neq \tilde{L}_{\mathcal{L}^{-} \rightarrow E}\right) \\
& \leq \limsup _{t \rightarrow \infty} \mathbb{P}\left(\max _{\kappa \in[0,1]}\left\{Y_{\left\lfloor\kappa\left(T+c_{-}(T) T^{2 / 3}\right)\right\rfloor}^{\mathrm{TOP}}-\kappa T\right\} \geq k T^{2 / 3} / 2\right) \\
& \leq C e^{-c k} .
\end{aligned}
$$

Finally, the independence of $\tilde{L}_{\mathcal{L}^{-} \rightarrow E}(k), \tilde{L}_{\mathcal{L}^{+} \rightarrow E^{+}}(k)$ follows from choosing $\varepsilon(a)>$ $k / a$ and (3.6): The admissible paths for $\tilde{L}_{\mathcal{L}^{+} \rightarrow E^{+}}(k)$ do not cross $R_{+}(k)$ from (3.12), and the admissible paths for $\tilde{L}_{\mathcal{L}^{-} \rightarrow E}(k)$ do not cross $R_{-}(k)$ from (3.14), and since $\varepsilon(a)>k / a$, we have by (3.6) that $R_{+}(k), R_{-}(k)$ do not cross each other, see also Figure 3.2. So $\tilde{L}_{\mathcal{L}^{-} \rightarrow E}(k), \tilde{L}_{\mathcal{L}^{+} \rightarrow E^{+}}(k)$ may only use points from disjoint, (deterministic) subsets of $\mathbb{Z}^{2}$, leading to the independence.

Proof of Theorem 2.4: Assumptions 1, 2, 3 of Theorem 2.8 have been verified in Propositions 3.1, 3.3, 3.4, such that the result follows.

Next we proof Theorem 2.1.

Proof of Theorem 2.1: Define $c_{1}=-\frac{\frac{u}{a}+a}{2}, c_{2}=\frac{u}{a}$ and $\xi_{2}=\frac{(u / a+a)^{2}}{2}-2^{-1 / 3} s$. Note that (see e.g. Theorem 5 in Cator and Pimentel, 2015) for $K \in \mathbb{N}, v \in \mathbb{R}, \gamma \in[0,1 / 3]$

$$
\lim _{K \rightarrow \infty} \frac{L_{0 \rightarrow\left(K+\left\lfloor K^{\gamma} v\right\rfloor, K\right)}-L_{0 \rightarrow(K, K)}-2 v K^{\gamma}}{K^{1 / 3}}=0 .
$$

In particular, since we are only interested in asymptotic results, any shift of order 1 of the end/ starting point for a point-to-point LPP time will be asymptotically irrelevant.We set

$$
t=\left\lfloor\frac{T}{4}+c_{1} T^{2 / 3}\right\rfloor \quad M=t+\left\lfloor c_{2} T^{2 / 3}+\xi_{2} T^{1 / 3}\right\rfloor
$$

Then $T^{1 / 3}=(4 t)^{1 / 3}+\mathcal{O}(1)$ as well as

$$
\begin{aligned}
& T=4 t-c_{1} t^{2 / 3} 4^{5 / 3}+c_{1}^{2} \frac{2}{3} t^{1 / 3} 4^{7 / 3}+\mathcal{O}(1) \\
& T^{2 / 3}=(4 t)^{2 / 3}-c_{1} \frac{2}{3} t^{1 / 3} 4^{4 / 3}+\mathcal{O}(1) .
\end{aligned}
$$

We define furthermore

$$
\hat{\mathcal{L}}^{+}=\left(\left\lfloor-a\left((4 t)^{2 / 3}-c_{1} \frac{2}{3} t^{1 / 3} 4^{4 / 3}\right)\right\rfloor, 0\right) \quad \hat{\mathcal{L}}^{-}=\left(0,\left\lfloor-a\left((4 t)^{2 / 3}-c_{1} \frac{2}{3} t^{1 / 3} 4^{4 / 3}\right)\right\rfloor\right)
$$

and $\hat{\mathcal{L}}=\hat{\mathcal{L}}^{+} \cup \hat{\mathcal{L}}^{-}$. Then by the link $(1.10)$

$$
\begin{aligned}
& \lim _{T \rightarrow \infty} \mathbb{P}\left(x_{\left\lfloor\frac{T}{4}-T^{2 / 3} \frac{a+\frac{u}{a}}{2}\right\rfloor}(T) \geq \frac{u}{a} T^{2 / 3}+T^{1 / 3} \frac{\left(\frac{u}{a}+a\right)^{2}}{2}-\frac{T^{1 / 3}}{2^{1 / 3}} s\right) \\
& =\lim _{T \rightarrow \infty} \mathbb{P}\left(L_{\left\{\left(-\left\lfloor a T^{2 / 3}\right\rfloor, 0\right),\left(0,-\left\lfloor a T^{2 / 3}\right\rfloor\right)\right\} \rightarrow\left(\left\lfloor\frac{T}{4}+c_{1} T^{2 / 3}+c_{2} T^{2 / 3}+\xi_{2} T^{1 / 3}\right\rfloor,\left\lfloor\frac{T}{4}+c_{1} T^{2 / 3}\right\rfloor\right)} \leq T\right) \\
& =\lim _{t \rightarrow \infty} \mathbb{P}\left(L_{\hat{\mathcal{L}} \rightarrow(M, t)} \leq 4 t-c_{1} t^{2 / 3} 4^{5 / 3}+c_{1}^{2} \frac{2}{3} t^{1 / 3} 4^{7 / 3}\right) .
\end{aligned}
$$


We now check the Assumptions 1, 2, 3 for the LPP times $L_{\hat{\mathcal{L}}^{+} \rightarrow(M, t)}$, $L_{\hat{\mathcal{L}}^{-} \rightarrow(M, t)}$. By Proposition 3.1 and (3.20), we have with $\mu_{\hat{\mathcal{L}}^{+} \rightarrow(M, t)}, \mu_{\hat{\mathcal{L}}^{-} \rightarrow(M, t)}$ defined by

$$
\begin{aligned}
\mu_{\hat{\mathcal{L}}^{+} \rightarrow(M, t)} t= & 4 t+2\left(c_{2}+a\right)(4 t)^{2 / 3}-4^{1 / 3}\left(c_{2}+a\right)^{2} t^{1 / 3} \\
& +2(4 t)^{1 / 3}\left(\xi_{2}-8 c_{1}\left(c_{2}+a\right) / 3\right) \\
\mu_{\hat{\mathcal{L}}^{-} \rightarrow(M, t)} t= & \mu_{\hat{\mathcal{L}}^{+} \rightarrow(M, t)} t+u 4^{4 / 3} t^{1 / 3}
\end{aligned}
$$

the convergence

$$
\begin{aligned}
& \lim _{t \rightarrow \infty} \mathbb{P}\left(L_{\hat{\mathcal{L}}^{+} \rightarrow(M, t)} \leq \mu_{\hat{\mathcal{L}}^{+} \rightarrow(M, t)} t+s 2^{4 / 3} t^{1 / 3}\right)=F_{\mathrm{GUE}}(s) \\
& \lim _{t \rightarrow \infty} \mathbb{P}\left(L_{\hat{\mathcal{L}}^{-} \rightarrow(M, t)} \leq \mu_{\hat{\mathcal{L}}^{-} \rightarrow(M, t)} t+s 2^{4 / 3} t^{1 / 3}\right)=F_{\mathrm{GUE}}(s) .
\end{aligned}
$$

The choice of $c_{1}, c_{2}, \xi_{2}$ is precisely such that

$$
\mu_{\hat{\mathcal{L}}^{+} \rightarrow(M, t)} t+s 2^{4 / 3} t^{1 / 3}=4 t-c_{1} t^{2 / 3} 4^{5 / 3}+c_{1}^{2} \frac{2}{3} t^{1 / 3} 4^{7 / 3} .
$$

So (3.25) verifies Assumption 1. Next we choose the point $\hat{E}^{+}$of Assumption 2. Note that with $\tilde{a}=a 4^{2 / 3}, \tilde{u}=4^{4 / 3} u$ we have

$$
L_{\hat{\mathcal{L}}^{+} \rightarrow(M, t)}=L_{\left(-\left\lfloor\tilde{a} t^{2 / 3}+\mathcal{O}\left(t^{1 / 3}\right)\right\rfloor, 0\right) \rightarrow\left(\left\lfloor t+\frac{\tilde{u}}{\tilde{a}} t^{2 / 3}+\mathcal{O}\left(t^{1 / 3}\right)\right\rfloor, t\right)} .
$$

This is, with $\tilde{a}, \tilde{u}$ instead of $a, u$ and up to an $\mathcal{O}\left(t^{1 / 3}\right)$ horizontal shift in the starting and end point, the same LPP time for which we chose $E^{+}$in (3.7). Hence we can take $\hat{E}^{+}$as $E^{+}$in (3.7), only with $\tilde{a}, \tilde{u}$ instead of $a, u$. Finally, Assumption 3 can be verified as in the proof of Theorem 2.4, the horizontal $\mathcal{O}\left(t^{1 / 3}\right)$ shifts in (3.27) (and in $L_{\hat{\mathcal{L}}^{-} \rightarrow(M, t)}$ ) not affecting the argument.

To prove Corollary 2.5, we need to know how the Airy ${ }_{2}$ arises in the LPP model of Theorem 2.4, hence the following lemma.

Lemma 3.5. Let $\mathcal{L}$ be as in Theorem 2.4. We have

$$
\begin{aligned}
\lim _{t \rightarrow \infty} \mathbb{P}\left(\frac{L_{\mathcal{L} \rightarrow\left(t+\frac{u}{a} t^{2 / 3}, t\right)}-\mu^{a} t}{2^{4 / 3} t^{1 / 3}} \leq s\right)=\mathbb{P}( & \mathcal{A}_{2}\left(\frac{-a-\frac{u}{a}}{2^{5 / 3}}\right) \leq s \\
& \left.\mathcal{A}_{2}\left(\frac{a-\frac{u}{a}}{2^{5 / 3}}\right) \leq s-\frac{u}{2^{4 / 3}}\right) .
\end{aligned}
$$

Proof: By exchanging the end point and $\mathcal{L}$ we see that

$$
L_{\mathcal{L} \rightarrow\left(t+\frac{u}{a} t^{2 / 3}, t\right)}={ }^{d} \max \left\{L_{(0,0) \rightarrow\left(t+(u / a+a) t^{2 / 3}, t\right)}, L_{(0,0) \rightarrow\left(t+u t^{2 / 3} / a, t+a t^{2 / 3}\right)}\right\}
$$

where $=^{d}$ denote equality in distribution. Now, for end points lying on the same horizontal line, the convergence of the rescaled LPP times to the $\mathcal{A}_{2}$ process is e.g. proven in Corollary 2.4 in Ferrari and Occelli (2018). To extend this result to the two endpoints of (3.30) (which do not lie on the same horizontal line), one follows the straight (characteristic) line joining $(0,0)$ and $\left(t+(u / a+a) t^{2 / 3}, t\right)$ until it reaches the horizontal line $\left(\cdot, t+a t^{2 / 3}\right)$ in a point $P$. By slow decorrelation, we may replace $L_{(0,0) \rightarrow\left(t+(u / a+a) t^{2 / 3}, t\right)}$ by $L_{(0,0) \rightarrow P}$ without altering the limiting distribution, giving the result. 
Proof of Corollary 2.5: It is an immediate Corollary of Theorem 2.4 and Lemma 3.5 , by a simple change of variable.

3.2. Proof of Theorems 2.7 and 2.8. Next we come to the proof of Theorem 2.7.

Proof of Theorem 2.7: The lower bound in (2.18) follows from the FKG inequality and the known convergence to the Airy 2 process, see Theorem 2 in Borodin and Péché (2008). For the upper bound, define the points

$$
P_{2}(u)=\left(\left\lfloor\tau t+u t^{2 / 3}\left(\tau a^{-1 / 3}-a^{2 / 3}\right)\right\rfloor,\lfloor\tau t+1\rfloor\right),
$$

and set $\mu_{u} t=4 \tau t+2 \tau t^{2 / 3} u a^{-1 / 3}-u^{2} \frac{\tau t^{1 / 3}}{4 a^{2 / 3}}$. Then for any $\delta>0$

$$
\lim _{t \rightarrow \infty} \mathbb{P}\left(\bigcup_{i=1}^{k} \frac{L_{P\left(a, u_{i}\right) \rightarrow P_{2}\left(u_{i}\right)}-\mu_{u_{i}} t}{2^{4 / 3}(a t)^{1 / 3}} \leq-\delta\right) \leq k F_{\mathrm{GUE}}\left(-\delta a^{1 / 3} \tau^{-1 / 3}\right) .
$$

Denote for brevity $\mathcal{F}=\bigcap_{i=1}^{l}\left\{L_{P\left(\tau, r_{i}\right) \rightarrow\left(\left\lfloor c t_{j}\right\rfloor,\left\lfloor\tau t_{j}\right\rfloor\right)}^{\text {resc }} \leq s_{i}\right\}$. Then, using subadditivity and (3.32), we get

$$
\begin{aligned}
& \lim _{t_{j} \rightarrow \infty} \mathbb{P}\left(\mathcal{F} \cap \bigcap_{i=1}^{k}\left\{L_{P\left(a, u_{i}\right) \rightarrow\left(\left\lfloor a t_{j}\right\rfloor,\left\lfloor a t_{j}\right\rfloor\right)}^{\mathrm{resc}} \leq \zeta_{i}\right\}\right) \\
& \leq \lim _{t_{j} \rightarrow \infty} \mathbb{P}\left(\mathcal { F } \cap \bigcap _ { i = 1 } ^ { k } \left\{\frac{L_{P\left(a, u_{i}\right) \rightarrow P_{2}\left(u_{i}\right)}-\mu_{u_{i}} t_{j}}{2^{4 / 3}\left(a t_{j}\right)^{1 / 3}}\right.\right. \\
& \left.\left.\quad+\frac{L_{P_{2}\left(u_{i}\right) \rightarrow\left(\left\lfloor a t_{j}\right\rfloor,\left\lfloor a t_{j}\right\rfloor\right)}-\mu\left(a, u_{i}\right) t_{j}+\mu_{u_{i}} t_{j}}{2^{4 / 3}\left(a t_{j}\right)^{1 / 3}} \leq \zeta_{i}\right\}\right) \\
& \leq \lim _{t_{j} \rightarrow \infty} \mathbb{P}\left(\mathcal{F} \cap \bigcap_{i=1}^{k}\left\{\frac{L_{P_{2}\left(u_{i}\right) \rightarrow\left(\left\lfloor a t_{j}\right\rfloor,\left\lfloor a t_{j}\right\rfloor\right)}-\mu\left(a, u_{i}\right) t_{j}+\mu_{u_{i}} t_{j}}{2^{4 / 3}\left(a t_{j}\right)^{1 / 3}} \leq \zeta_{i}+\delta\right\}\right) \\
& +k F_{\mathrm{GUE}}\left(-\delta a^{1 / 3} \tau^{-1 / 3}\right) .
\end{aligned}
$$

Note now that $L_{P(\tau, r) \rightarrow(\lfloor\tau t\rfloor,\lfloor\tau t\rfloor)}$ and $L_{P_{2}(u) \rightarrow(\lfloor a t\rfloor,\lfloor a t\rfloor)}$ are independent for all $r, u \in$ $\mathbb{R}$. Hence we get that

$$
\begin{aligned}
(3.34) & =\lim _{t \rightarrow \infty} \mathbb{P}(\mathcal{F}) \mathbb{P}\left(\bigcap_{i=1}^{k}\left\{\frac{L_{P_{2}\left(u_{i}\right) \rightarrow(\lfloor a t\rfloor,\lfloor a t\rfloor)}-\mu\left(a, u_{i}\right) t+\mu_{u_{i}} t}{2^{4 / 3}(a t)^{1 / 3}} \leq \zeta_{i}+\delta\right\}\right) \\
& =\mathbb{P}\left(\bigcap_{i=1}^{l} \mathcal{A}_{2}\left(r_{i}\right) \leq s_{i}\right) \mathbb{P}\left(\bigcap_{i=1}^{k} \mathcal{A}_{2}\left(u_{i}(1-\tau / a)^{1 / 3}\right) \leq\left(\zeta_{i}+\delta\right) \frac{a^{1 / 3}}{(a-\tau)^{1 / 3}}\right),
\end{aligned}
$$

finishing the proof.

We conclude by proving the general Theorem 2.8 .

Proof of Theorem 2.8: For the lower bound, note that $\left\{L_{\mathcal{L}^{+} \rightarrow E_{1}}^{\text {resc }} \leq s_{1}\right\}$, $\left\{L_{\mathcal{L}^{-} \rightarrow E_{2}}^{\text {resc }} \leq s_{2}\right\}$ are decreasing events. Thus by the FKG inequality we have

$$
\mathbb{P}\left(L_{\mathcal{L}^{+} \rightarrow E_{1}}^{\text {resc }} \leq s_{1}\right) \mathbb{P}\left(L_{\mathcal{L}^{-} \rightarrow E_{2}}^{\text {resc }} \leq s_{2}\right) \leq \mathbb{P}\left(L_{\mathcal{L}^{+} \rightarrow E_{1}}^{\text {resc }} \leq s_{1}, L_{\mathcal{L}^{-} \rightarrow E_{2}}^{\text {resc }} \leq s_{2}\right) .
$$


Now by Assumption 1 we have

$$
\lim _{t \rightarrow \infty} \mathbb{P}\left(L_{\mathcal{L}^{+} \rightarrow E_{1}}^{\text {resc }} \leq s_{1}\right) \mathbb{P}\left(L_{\mathcal{L}^{-} \rightarrow E_{2}}^{\text {resc }} \leq s_{2}\right)=G_{1}^{a}\left(s_{1}\right) G_{2}^{a}\left(s_{2}\right)
$$

which proves the lower bound.

For the upper bound, denote $A^{\delta}=\left\{L_{E^{+} \rightarrow E_{1}}^{\text {resc }} \leq-\delta\right\}$. Noting that $L_{\mathcal{L}^{+} \rightarrow E_{1}}^{\text {resc }} \geq$ $L_{E^{+} \rightarrow E_{1}}^{\text {resc }}+L_{\mathcal{L}^{+} \rightarrow E^{+}}^{\text {resc }}$ we get from Assumptions 1, 2 and 3

$$
\begin{aligned}
& \lim _{t_{k} \rightarrow \infty} \mathbb{P}\left(L_{\mathcal{L}^{+} \rightarrow E_{1}}^{\text {resc }} \leq s_{1}, L_{\mathcal{L}^{-} \rightarrow E_{2}}^{\text {resc }} \leq s_{2}\right) \\
& \leq \lim _{t_{k} \rightarrow \infty} \mathbb{P}\left(\left\{L_{E^{+} \rightarrow E_{1}}^{\text {resc }}+L_{\mathcal{L}^{+} \rightarrow E^{+}}^{\text {resc }} \leq s_{1}\right\} \cap\left\{L_{\mathcal{L}^{-} \rightarrow E_{2}}^{\text {resc }} \leq s_{2}\right\} \cap\left(A^{\delta} \cup\left(A^{\delta}\right)^{c}\right)\right) \\
& \leq G_{0}^{a}(-\delta)+\lim _{t_{k} \rightarrow \infty} \mathbb{P}\left(\left\{-\delta+L_{\mathcal{L}^{+} \rightarrow E+}^{\text {resc }} \leq s_{1}\right\} \cap\left\{L_{\mathcal{L}^{-} \rightarrow E_{2}}^{\text {resc }} \leq s_{2}\right\} \cap\left(A^{\delta}\right)^{c}\right) \\
& \leq G_{0}^{a}(-\delta)+\lim _{t_{k} \rightarrow \infty} \mathbb{P}\left(\left\{L_{\mathcal{L}^{+} \rightarrow E^{+}}^{\text {resc }} \leq s_{1}+\delta\right\} \cap\left\{L_{\mathcal{L}^{-} \rightarrow E_{2}}^{\text {resc }} \leq s_{2}\right\}\right) \\
& \leq G_{0}^{a}(-\delta)+\tilde{\psi}+\lim _{t_{k} \rightarrow \infty} \mathbb{P}\left(\left\{\tilde{L}_{\mathcal{L}^{+} \rightarrow E^{+}}^{\text {resc }} \leq s_{1}+\delta\right\}\right) \mathbb{P}\left(\left\{\tilde{L}_{\mathcal{L}^{-} \rightarrow E_{2}}^{\text {resc }} \leq s_{2}\right\}\right) \\
& \leq G_{0}^{a}(-\delta)+3 \tilde{\psi}+\lim _{t_{k} \rightarrow \infty} \mathbb{P}\left(\left\{L_{\mathcal{L}^{+} \rightarrow E^{+}}^{\text {resc }} \leq s_{1}+\delta\right\}\right) \mathbb{P}\left(\left\{L_{\mathcal{L}^{-} \rightarrow E_{2}}^{\text {resc }} \leq s_{2}\right\}\right) \\
& =G_{0}^{a}(-\delta)+3 \tilde{\psi}+G_{1}^{a}\left(\left(s_{1}+\delta\right) c_{\varepsilon(a)}\right) G_{2}^{a}\left(s_{2}\right) .
\end{aligned}
$$

\section{Proof for line-to-point problems: Theorems 2.2 and 2.6}

In this Section we prove Theorem 2.2 as well as Theorem 2.6.

We also discuss the transition to shock fluctuations when for $a=0$ one has flat (deterministic) initial data.

We start with the proof of Theorem 2.6. The proof is summarized in Figure 2, the actual proof is slightly technical.

Proof of Theorem 2.6: We start by proving (2.12). We apply Theorem 2.8 with $\mathcal{L}=\mathcal{L}^{+}=\mathcal{L}^{-}=\{(-k, k): k \in \mathbb{Z}\}, E_{+}=E_{1}=(\lfloor t\rfloor,\lfloor t\rfloor), E_{2}=\left(\lfloor t\rfloor-\left\lfloor a t^{2 / 3}\right\rfloor,\lfloor t\rfloor+\right.$ $\left.\left\lfloor a t^{2 / 3}\right\rfloor\right)$. One obtains from Theorem 2.2 of Borodin and Ferrari (2008) and the link between TASEP and LPP (all weights i.i.d., $\omega_{i, j} \sim \exp (1)$ )

$$
\lim _{t \rightarrow \infty} \mathbb{P}\left(\cap_{i=1}^{2}\left\{L_{\mathcal{L} \rightarrow E_{i}} \leq 4 t+s_{i} t^{1 / 3}\right\}\right)=\mathbb{P}\left(\mathcal{A}_{1}(0) \leq 2^{-5 / 3} s_{1}, \mathcal{A}_{1}\left(a 4^{-2 / 3}\right) \leq 2^{-5 / 3} s_{2}\right) .
$$

Also for $i=1,2$ we have

$$
\lim _{t \rightarrow \infty} \mathbb{P}\left(L_{\mathcal{L} \rightarrow E_{i}} \leq 4 t+s t^{1 / 3}\right)=F_{\mathrm{GOE}}\left(2^{-2 / 3} s\right) .
$$

Now (4.2) shows Assumption 1, and Assumption 2 is trivially fullfilled with $c_{\varepsilon(a)}=$ $1, G_{0}^{a}=\mathbf{1}_{[0, \infty)}$. Set now for $k \in \mathbb{Z}$

$$
\begin{aligned}
& \mathcal{F}^{1}(k)=\left\{(-i, i), i=-\left\lfloor k t^{2 / 3}\right\rfloor, \ldots,\left\lfloor k t^{2 / 3}\right\rfloor\right\}, \\
& \mathcal{F}^{2}(k)=\left\{\left(-\left\lfloor a t^{2 / 3}\right\rfloor-i,\left\lfloor a t^{2 / 3}\right\rfloor+i\right), i=-\left\lfloor k t^{2 / 3}\right\rfloor, \ldots,\left\lfloor k t^{2 / 3}\right\rfloor\right\} .
\end{aligned}
$$

Denote by $\pi_{\mathcal{L} \rightarrow E_{i}}^{\max }$ the maximizing path from $\mathcal{L}$ to $E_{i}$ and by $\pi_{\mathcal{L} \rightarrow E_{i}}^{\max }(0)$ the point of $\pi_{\mathcal{L} \rightarrow E_{i}}$ which belongs to $\mathcal{L}$. By a simple shift one sees $\mathbb{P}\left(\pi_{\mathcal{L} \rightarrow E_{i}}^{\max }(0) \in \mathcal{F}^{i}(k)\right)$ is the same for $i=1,2$. Consequently, by (4.18) of Ferrari and Occelli (2018) one gets that for $k$ sufficiently large and some constants $C, c$

$$
\mathbb{P}\left(\cup_{i=1}^{2}\left\{\pi_{\mathcal{L} \rightarrow E_{i}}^{\max }(0) \notin \mathcal{F}^{i}(k)\right\}\right) \leq C e^{-c k^{2}} .
$$




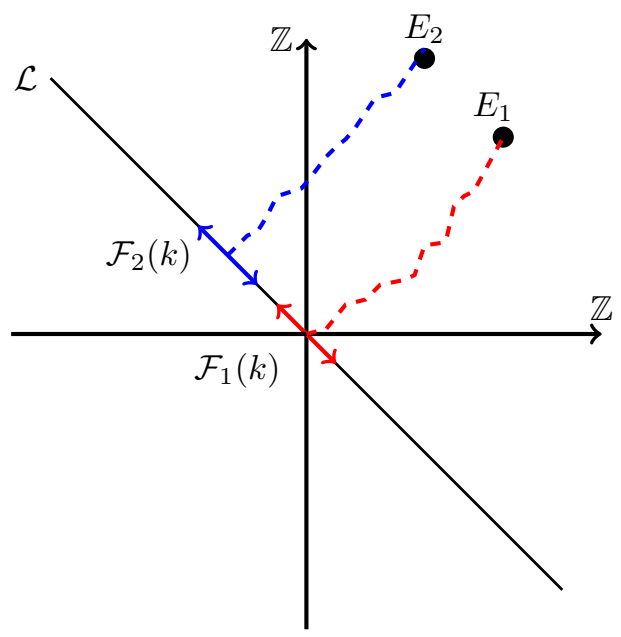

Figure 4.3. Proving (2.12): The maximizer (blue, dashed) $\pi_{\mathcal{L} \rightarrow E_{2}}$ from $\mathcal{L}=\{(-k, k): k \in \mathbb{Z}\}$ to $E_{2}=\left(t-a t^{2}, t+a t^{2 / 3}\right)$ starts with high probability in the line segment $F_{2}(k)$ (blue, see (4.4)). In this event, the transversal fluctuations of $\pi_{\mathcal{L} \rightarrow E_{2}}^{\max }$ are bounded by those of the maximizer starting at the bottom right end point of $F_{2}(k)$ and going to $E_{2}$, which themselves are bounded by Theorem 3.2. The same argument applies to $\pi_{\mathcal{L} \rightarrow E_{1}}^{\max }$ (red,dashed) with $E_{1}=(t, t)$. This shows $\pi_{\mathcal{L} \rightarrow E_{1}}^{\max }, \pi_{\mathcal{L} \rightarrow E_{2}}^{\max }$ stay in disjoint sets with high probability, which together with convergence to the Airy process of the LPP time, implies (2.12).

Let $E_{3}=\left(-\left\lfloor\frac{a}{4} t^{2 / 3}\right\rfloor,\left\lfloor\frac{a}{4} t^{2 / 3}\right\rfloor\right)$ and $E_{4}=E_{3}+E_{1}$. Define also $E_{5}=\left(-\left\lfloor\frac{3 a}{4} t^{2 / 3}\right\rfloor,\left\lfloor\frac{3 a}{4} t^{2 / 3}\right\rfloor\right), E_{6}=E_{5}+E_{1}$. Denote by $R_{1}(k)$ the straight line (in $\mathbb{Z}^{2}$ ) which goes through $E_{3}+\left(-\left\lfloor k t^{2 / 3}\right\rfloor,\left\lfloor k t^{2 / 3}\right\rfloor\right)$ and $E_{4}+\left(-\left\lfloor k t^{2 / 3}\right\rfloor,\left\lfloor k t^{2 / 3}\right\rfloor\right)$ and by $R_{2}(k)$ the straight line going through $E_{5}+\left(\left\lfloor k t^{2 / 3}\right\rfloor,-\left\lfloor k t^{2 / 3}\right\rfloor\right)$ and $E_{6}+$ $\left(\left\lfloor k t^{2 / 3}\right\rfloor,-\left\lfloor k t^{2 / 3}\right\rfloor\right)$. Denote by $U_{1}$ the event $\left\{\pi_{E_{3} \rightarrow E_{4}}^{\max } \cap R_{1}(a / 10)=\emptyset\right\}$ and by $U_{2}$ the event $\left\{\pi_{E_{5} \rightarrow E_{6}}^{\max } \cap R_{2}(a / 10)=\emptyset\right\}$.

It follows from Theorem 3.2 that

$$
\limsup _{t \rightarrow \infty} \mathbb{P}\left(\cup_{i=1}^{2} U_{i}^{c}\right) \leq C e^{-c a} .
$$

Now the event

$$
\bigcap_{i=1}^{2}\left\{\pi_{\mathcal{L} \rightarrow E_{i}}^{\max }(0) \in \mathcal{F}^{i}(a / 10)\right\} \cap \bigcap_{i=1}^{2} U_{i}
$$

is a subset of

$$
\bigcap_{i=1}^{2}\left\{\pi_{\mathcal{L} \rightarrow E_{i}}^{\max } \cap R_{i}(a / 10)=\emptyset\right\}
$$

(taking $a, t$ sufficiently large). Denote now for $i=1,2$ by $\Pi^{i}$ the set of up-right paths from $\mathcal{L}$ to $E_{i}$ which contain no point of $R_{i}(a / 10)$. Set

$$
\tilde{L}_{\mathcal{L} \rightarrow E_{i}}=\max _{\pi \in \Pi^{i}} \sum_{(i, j) \in \pi} \omega_{i, j} .
$$


Note that $\tilde{L}_{\mathcal{L} \rightarrow E_{i}}, i=1,2$ are independent, and by (4.5), (4.6) Assumption 3 is fullfilled with

$$
\tilde{\psi}=\tilde{\psi}(a)=C e^{-c a^{2}}+C e^{-c a} .
$$

This finishes the proof.

Next we come to the proof of (2.15). Set $\mathcal{L}^{\text {half }}=\{(-k, k): k \geq 0\}$. It follows from Theorem 2 of Borodin et al. (2008) that with $E(k)=\left(\left\lfloor t-k t^{2 / 3}\right\rfloor,\left\lfloor t+k t^{2 / 3}\right\rfloor\right)$ and $b_{1}, b_{2} \in \mathbb{R}$

$$
\begin{aligned}
& \lim _{t \rightarrow \infty} \mathbb{P}\left(\cap_{i=1}^{2}\left\{L_{\mathcal{L}^{\text {half }} \rightarrow E\left(b_{i}\right)} \leq 4 t+\left(s_{i}-2^{4 / 3} \min \left\{0, b_{i}\right\}^{2}\right) t^{1 / 3}\right\}\right) \\
& =\mathbb{P}\left(\mathcal{A}_{2 \rightarrow 1}\left(b_{1} 2^{-2 / 3}\right) \leq 2^{-4 / 3} s_{1}, \mathcal{A}_{2 \rightarrow 1}\left(b_{2} 2^{-2 / 3}\right) \leq 2^{-4 / 3} s_{2}\right)
\end{aligned}
$$

To localize $\pi_{\mathcal{L}^{\text {half }} \rightarrow E(|b|+a)}^{\max }(0)$ note that by a simple coupling, with $E_{7}=(-\lfloor(|b|+$ a) $\left.\left.t^{2 / 3}\right\rfloor,\left\lfloor(|b|+a) t^{2 / 3}\right\rfloor\right)$ and

$$
\mathcal{F}^{3}(k)=\left\{E_{7}+(-i, i): i=-\left\lfloor k t^{2 / 3}\right\rfloor, \ldots,\left\lfloor k t^{2 / 3}\right\rfloor\right\}
$$

we have $\left\{\pi_{\mathcal{L} \rightarrow E(|b|+a)}^{\max }(0) \in \mathcal{F}^{3}(a / 10)\right\} \subseteq\left\{\pi_{\mathcal{L}^{\text {half }} \rightarrow E(|b|+a)}^{\max }(0) \in \mathcal{F}^{3}(a / 10)\right\}$ such that $\mathbb{P}\left(\left\{\pi_{\mathcal{L}^{\text {half }} \rightarrow E(|b|+a)}^{\max }(0) \notin \mathcal{F}^{3}(a / 10)\right\}\right) \leq C e^{-c a^{2}}$ by (4.18) in Ferrari and Occelli (2018). Similarily, one can control $\mathbb{P}\left(\pi_{\mathcal{L}^{\text {half }} \rightarrow E(|b|+a / 5)}^{\max }(0) \notin \mathcal{F}^{4}(a / 20)\right) \leq$ $C e^{-c a^{2}}$, where $\mathcal{F}^{4}(k)=\left\{E_{8}+(-i, i): i=-\left\lfloor k t^{2 / 3}\right\rfloor, \ldots,\left\lfloor k t^{2 / 3}\right\rfloor\right\}$ with $E_{8}=$ $\left(-\left\lfloor(|b|+a / 5) t^{2 / 3}\right\rfloor,\left\lfloor(|b|+a / 5) t^{2 / 3}\right\rfloor\right)$. Let $R_{3}(k)$ be the line which connects $E_{8}+$ $\left(-\left\lfloor k t^{2 / 3}\right\rfloor,\left\lfloor k t^{2 / 3}\right\rfloor\right)$ with $E(|b|+a / 5)+\left(-\left\lfloor k t^{2 / 3}\right\rfloor,\left\lfloor k t^{2 / 3}\right\rfloor\right)$ and $R_{4}(k)$ the line which connects $E_{7}+\left(\left\lfloor k t^{2 / 3}\right\rfloor,-\left\lfloor k t^{2 / 3}\right\rfloor\right)$ with $E(|b|+a)+\left(\left\lfloor k t^{2 / 3}\right\rfloor,-\left\lfloor k t^{2 / 3}\right\rfloor\right)$. As was done above, we can bound

$$
\begin{aligned}
& \mathbb{P}\left(\pi_{\mathcal{L}^{\text {half }} \rightarrow E(|b|+a / 5)}^{\max } \cap R_{3}(a / 10) \neq \emptyset\right) \leq C e^{-c a^{2}}+C e^{-c a} \\
& \mathbb{P}\left(\pi_{\mathcal{L}^{\text {half }} \rightarrow E(|b|+a)}^{\max } \cap R_{4}(a / 10) \neq \emptyset\right) \leq C e^{-c a^{2}}+C e^{-c a} .
\end{aligned}
$$

Note now that if $\pi_{\mathcal{L}^{\text {half }} \rightarrow E(|b|+a / 5)}^{\max }$ contains no point of $R_{3}(k)$ then this is also true for $\pi_{\mathcal{L}^{\text {half }} \rightarrow E(b)}^{\max }$. Let $\Pi^{3}$ be the up-right paths from $\mathcal{L}^{\text {half }}$ to $E(b)$ which contain no point of $R_{3}(a / 10)$, and $\Pi^{4}$ be the up-right paths from $\mathcal{L}^{\text {half }}$ to $E(|b|+a)$ which contain no point of $R_{4}(a / 10)$. We define the independent random variables

$$
\tilde{L}_{\mathcal{L}^{\text {half }} \rightarrow E(b)}=\max _{\pi \in \Pi^{3}} \sum_{(i, j) \in \pi} \omega_{i, j} \quad \tilde{L}_{\mathcal{L}^{\text {half }} \rightarrow E(|b|+a)}=\max _{\pi \in \Pi^{4}} \sum_{(i, j) \in \pi} \omega_{i, j} .
$$

Now we take $\mathcal{L}^{\text {half }}=\mathcal{L}^{+}=\mathcal{L}^{-}, E_{1}=E_{+}=E(b), E_{2}=E(|b|+a)$. Then Assumption 1 holds by (4.11), and Assumption 2 holds trivially with $c_{\varepsilon(a)}=1, G_{0}^{a}=\mathbf{1}_{[0, \infty)}$. Finally, by (4.12), Assumption 3 holds with $\tilde{\psi}$ as in (4.10).

Next we come to the proof of Theorem 2.2. Here we need to use slow decorrelation again, as we have two maximizing paths going to the same endpoint, on the other hand, the proof is easier since the two maximizers start at points at distance $\mathcal{O}(T)$ from each other (see (4.18)), not $\mathcal{O}\left(T^{2 / 3}\right)$ as in Theorems 2.4, 2.6.

Proof of Theorem 2.2: We work in the last passage picture, where Theorem 2.2 is equivalent to

$$
\lim _{T \rightarrow \infty} \mathbb{P}\left(L_{\mathcal{L}^{\varrho_{1}} \cup \mathcal{L}^{\varrho_{2}} \rightarrow E} \leq T\right)=F_{\mathrm{GOE}}\left(2^{2 / 3}\left(s-\xi / \varrho_{1}\right) c_{1}\right) F_{\mathrm{GOE}}\left(2^{2 / 3}\left(s-\xi / \varrho_{2}\right) c_{2}\right),
$$


where we defined

$$
\begin{aligned}
& E=\left(\left(1-\varrho_{1}-\varrho_{2}+\varrho_{1} \varrho_{2}\right) T-(s-\xi) T^{1 / 3}, \varrho_{1} \varrho_{2} T+\xi T^{1 / 3}\right) \\
& \mathcal{L}^{\varrho_{1}}=\left\{\left(n-\left\lfloor n / \varrho_{1}\right\rfloor, n\right), n \leq 0\right\} \quad \mathcal{L}^{\varrho_{2}}=\left\{\left(n-\left\lfloor n / \varrho_{2}\right\rfloor, n\right), n>0\right\} .
\end{aligned}
$$

Denote by $\pi_{\mathcal{L}^{\varrho_{i} \rightarrow E}}^{\max }, i=1,2$ the maximizing paths from $\mathcal{L}^{\varrho_{i}} \rightarrow E$. Consider the points

$$
S_{\varrho_{1}}=\left(\left(1-\varrho_{1}\right)\left(\varrho_{1}-\varrho_{2}\right), \varrho_{1}\left(\varrho_{2}-\varrho_{1}\right)\right) T \quad S_{\varrho_{2}}=\left(\left(1-\varrho_{2}\right)\left(\varrho_{2}-\varrho_{1}\right),\left(\varrho_{1}-\varrho_{2}\right) \varrho_{2}\right) T .
$$

The lines $\overline{S_{\varrho_{i}} E}$ are the characteristic lines of $\pi_{\mathcal{L}^{\varrho_{i}} \rightarrow E}^{\max }$. Note that

$$
\left\|S_{\varrho_{1}}-S_{\varrho_{2}}\right\|_{2}=\mathcal{O}\left(\left(\varrho_{1}-\varrho_{2}\right) T\right) .
$$

That (4.18) is of order $T$ (and not $T^{2 / 3}$ ) is the main difference to all other situations considered in this paper, and implies that $L_{\mathcal{L} \varrho_{1} \rightarrow E}, L_{\mathcal{L} \varrho_{2} \rightarrow E}$ decouple already in the $T \rightarrow \infty$ limit. Define the point

$$
E^{\varrho_{2}}=E-\left(\left(1-\varrho_{2}\right)^{2} T^{\nu}, \varrho_{2}^{2} T^{\nu}\right), \nu \in(2 / 3,1),
$$

which lies on $\overline{S_{\varrho_{2}} E}$. We can then localize the starting point of $\pi_{\mathcal{L}^{\varrho_{2}} \rightarrow E^{\varrho_{2}}}^{\max }$ by $(4.18)$ of Ferrari and Occelli (2018), which gives that

$$
H_{2}=\left\{\pi_{\mathcal{L}^{\varrho_{2}} \rightarrow E^{\varrho_{2}}}^{\max }(0) \in\left\{P \in \mathcal{L}^{\varrho_{2}}:\left\|P-S_{\varrho_{2}}\right\|_{2} \leq k T^{2 / 3}\right\}\right\}
$$

has probability $\mathbb{P}\left(H_{2}\right)>1-e^{c k^{2}}$ for $T$, a large enough. For our purposes it suffices to know that for any $\varepsilon \in(0,1 / 3)$ we have that

$$
\tilde{H}_{2}=\left\{\pi_{\mathcal{L}^{\varrho_{2}} \rightarrow E^{\varrho_{2}}}^{\max }(0) \in\left\{P \in \mathcal{L}^{\varrho_{2}}:\left\|P-S_{\varrho_{2}}\right\|_{2} \leq T^{2 / 3+\varepsilon}\right\}\right\}
$$

satisfies $\mathbb{P}\left(\tilde{H}_{2}\right) \rightarrow_{T \rightarrow \infty}$ 1. Exactly as in the proof of Theorem 2.6, on the event $\tilde{H}_{2}$, we can bound the transversal fluctuations of $\pi_{\mathcal{L}^{\varrho_{2}} \rightarrow E^{\varrho^{2}}}^{\max }$ by the transversal fluctuations of $\pi_{P^{\prime} \rightarrow E^{\varrho_{2}}}$ where $P^{\prime}$ is the bottom right end point of the line segment $\left\{P \in \mathcal{L}^{\varrho_{2}}:\left\|P-S_{\varrho_{2}}\right\|_{2} \leq T^{2 / 3+\varepsilon}\right\}:$ By Theorem 3.2, the transversal fluctuations of $\pi_{P^{\prime} \rightarrow E^{\varrho_{2}}}^{\max }$ around $\overline{P^{\prime} E^{\varrho_{2}}}$ are $o\left(T^{2 / 3+\varepsilon}\right)$ for any $\varepsilon>0$.

We can localize the starting point $\pi_{\mathcal{L} e_{1} \rightarrow E}^{\max }(0)$ in exactly the same way, as well as the transversal fluctuations of $\pi_{\mathcal{L}^{\ell_{1}} \rightarrow E}^{\max }$. If we choose $1>\nu>2 / 3+\varepsilon$, then we have shown there are disjoint deterministic sets $D_{\varrho_{1}}, D_{\varrho_{2}}$, such that

$$
\lim _{T \rightarrow \infty} \mathbb{P}\left(\pi_{\mathcal{L}^{\varrho_{2}} \rightarrow E \varrho_{2}}^{\max } \subset D_{\varrho_{2}}, \pi_{\mathcal{L}^{\varrho_{1}} \rightarrow E}^{\max } \subset D_{\varrho_{1}}\right)=1 \text {. }
$$

This implies

$$
\begin{aligned}
& \lim _{T \rightarrow \infty} \mathbb{P}\left(L_{\mathcal{L} \varrho_{1} \cup \mathcal{L} \varrho_{1} \rightarrow E} \leq T\right)=\lim _{T \rightarrow \infty} \mathbb{P}\left(L_{\mathcal{L} \varrho_{1} \rightarrow E} \leq T, L_{\mathcal{L} \varrho_{2} \rightarrow E} \leq T\right) \\
&=\lim _{T \rightarrow \infty} \mathbb{P}\left(L_{\mathcal{L}} \varrho_{1} \rightarrow E \leq T\right) \mathbb{P}\left(L_{\mathcal{L}} \leq \varrho_{2} \rightarrow E\right. \\
&
\end{aligned}
$$

(the first identity holds by definition, the second by (4.22) and slow decorrelation, which implies that $T^{-1 / 3}\left(L_{\mathcal{L}^{\varrho_{2}} \rightarrow E^{\varrho_{2}}}+\mu_{0} T^{\nu}-L_{\mathcal{L}^{\varrho_{2}} \rightarrow E}\right)$ converges to 0 in probability for the correct $\left.\mu_{0}\right)$. So what remains to compute is

$$
\begin{aligned}
\lim _{T \rightarrow \infty} \mathbb{P}\left(L_{\mathcal{L} \varrho_{1} \rightarrow E} \leq T\right) \mathbb{P} & \left(L_{\mathcal{L} \varrho_{2} \rightarrow E} \leq T\right) \\
& =F_{\mathrm{GOE}}\left(2^{2 / 3}\left(s-\xi / \varrho_{1}\right) c_{1}\right) F_{\mathrm{GOE}}\left(2^{2 / 3}\left(s-\xi / \varrho_{2}\right) c_{2}\right),
\end{aligned}
$$

which has, (with the $\mathcal{O}\left(T^{1 / 3}\right)$ terms taken from $E$ into the scaling), been proven in Lemma 2.4 of Ferrari et al. $(2018+)$. 
Now let us come to the statement (2.10). We follow the proof of Theorem 2.2. We choose the point

$\tilde{E}=\left(\left(1-\varrho_{1}(a)-\varrho_{2}+\varrho_{1}(a) \varrho_{2}\right) T-\xi T^{2 / 3}\left(\frac{1}{a \varrho_{2}}-\frac{1}{a}\right)-s T^{1 / 3} / c_{2}, \varrho_{1}(a) \varrho_{2} T+\xi T^{2 / 3} / a\right)$.

The first difference arises in (4.14): It is unclear what

$$
\lim _{T \rightarrow \infty} \mathbb{P}\left(L_{\mathcal{L}^{\varrho_{1}(a)} \cup \mathcal{L}^{\varrho_{2}} \rightarrow \tilde{E}} \leq T\right)
$$

is, and a priori it is even unclear whether (4.28) exists as a limit in distribution. Knowing this is however not needed to study the $a \rightarrow \infty$ asymptotics, we may simply consider an arbitrary subsequential limit $T_{k} \rightarrow \infty$ and show that we have

$$
\begin{aligned}
\lim _{a \rightarrow+\infty} \lim _{T_{k} \rightarrow \infty} & \mathbb{P}\left(L_{\mathcal{L}^{\varrho_{1}(a)} \cup \mathcal{L}^{\varrho_{2}} \rightarrow \tilde{E}} \leq T_{k}\right) \\
& =F_{\mathrm{GOE}}\left(2^{2 / 3} s\right) F_{\mathrm{GOE}}\left(2^{2 / 3} s-2^{2 / 3} \xi\left(1-\varrho_{2}\right)^{-2 / 3} \varrho_{2}^{-5 / 3}\right) .
\end{aligned}
$$

Now to show (4.29), we again need to choose the point $E^{\varrho_{2}}$ from (4.19), but as in the proof of Theorem 2.4, $E^{\varrho_{2}}$ needs to have distance $\mathcal{O}(T)$ from $\tilde{E}$, we may choose $E^{\varrho_{2}}=\tilde{E}-\left(\left(1-\varrho_{2}\right)^{2} T a^{-1 / 2}, \varrho_{2}^{2} T a^{-1 / 2}\right)$. With this choice, we have an extended slow decorrelation result in the double limit $\lim _{a \rightarrow+\infty} \lim _{T \rightarrow \infty}$.

Next note that the maximizers $\pi_{\mathcal{L}^{\varrho_{2}} \rightarrow E^{\varrho_{2}}}^{\max }, \pi_{\mathcal{L}^{\varrho_{1}(a)} \rightarrow \tilde{E}}^{\max }$ start in $\mathcal{O}\left(T^{2 / 3}\right)$ neighborhoods of the points $S_{\varrho_{1}(a)}, S_{\varrho_{2}}$ (defined in (4.17)), which themselves have distance $C a T^{2 / 3}+o\left(T^{2 / 3}\right), C>0$, from each other (see (4.18)). Doing a refined localization of the starting points as in (4.20) with e.g. $k=a^{1 / 10}$, and bounding the transversal fluctuations, we see that there are disjoint deterministic sets $\tilde{D}_{\varrho_{1}(a)}, \tilde{D}_{\varrho_{2}}$ such that

$$
\lim _{a \rightarrow+\infty} \liminf _{T \rightarrow \infty} \mathbb{P}\left(\pi_{\mathcal{L}^{\varrho_{2} \rightarrow E \varrho_{2}}}^{\max } \subset \tilde{D}_{\varrho_{2}}, \pi_{\mathcal{L} \varrho_{1}(a) \rightarrow \tilde{E}}^{\max } \subset \tilde{D}_{\varrho_{1}(a)}\right)=1,
$$

which implies

$$
\begin{array}{r}
\lim _{a \rightarrow+\infty} \lim _{T_{k} \rightarrow \infty} \mathbb{P}\left(L_{\mathcal{L}^{\varrho_{1}(a)} \cup \mathcal{L}^{\varrho_{2}} \rightarrow \tilde{E}} \leq T_{k}\right)=\lim _{a \rightarrow+\infty} \lim _{T_{k} \rightarrow \infty} \mathbb{P}\left(L_{\mathcal{L}^{\varrho_{1}(a)} \rightarrow \tilde{E}} \leq T_{k}\right) \\
\times \mathbb{P}\left(L_{\mathcal{L}^{\varrho_{2}} \rightarrow \tilde{E}} \leq T_{k}\right) .
\end{array}
$$

Finally, we need to show that the individual $\mathbb{P}\left(L_{\mathcal{L}^{\varrho_{1}(a)} \rightarrow \tilde{E}} \leq T\right), \mathbb{P}\left(L_{\mathcal{L}^{\varrho_{2}} \rightarrow \tilde{E}} \leq T\right)$ converge to $F_{\mathrm{GOE}}$ in the double limit $\lim _{a \rightarrow+\infty} \lim _{T \rightarrow \infty}$. The localization of the starting point shows that when considering the full lines $\mathcal{L}^{\varrho_{1}(a), \infty}=\{(n-$ $\left.\left.\left\lfloor n / \varrho_{1}(a)\right\rfloor, n\right), n \in \mathbb{Z}\right\} \quad \mathcal{L}^{\varrho_{2}, \infty}=\left\{\left(n-\left\lfloor n / \varrho_{2}\right\rfloor, n\right), n \in \mathbb{Z}\right\}$ we have that

$$
\lim _{a \rightarrow+\infty} \limsup _{T \rightarrow \infty} \mathbb{P}\left(\left\{L_{\mathcal{L}^{\varrho_{1}(a)} \rightarrow \tilde{E}} \neq L_{\mathcal{L}^{\varrho_{1}(a), \infty} \rightarrow \tilde{E}}\right\} \cup\left\{L_{\mathcal{L}^{\varrho_{2}} \rightarrow \tilde{E}} \neq L_{\mathcal{L}^{\varrho_{2}, \infty} \rightarrow \tilde{E}}\right\}\right)=0 .
$$

Now the convergence, for $a$ fixed, of the properly rescaled $L_{\mathcal{L} \varrho_{1}(a), \infty \rightarrow \tilde{E}}, L_{\mathcal{L} \varrho_{2}, \infty \rightarrow \tilde{E}}$ to $F_{\mathrm{GOE}}$ should be deduced from Theorem 2.1 of Ferrari and Occelli (2018). This would then prove (4.29).

\section{References}

M. Adler and P. van Moerbeke. PDEs for the joint distributions of the Dyson, Airy and Sine processes. Ann. Probab. 33 (4), 1326-1361 (2005). MR2150191.

J. Baik and Z. Liu. Multi-point distribution of periodic TASEP. ArXiv Mathematics e-prints (2018). arXiv: 1710.03284. 
M. Balázs, E. Cator and T. Seppäläinen. Cube root fluctuations for the corner growth model associated to the exclusion process. Electron. J. Probab. 11, no. 42, 1094-1132 (2006). MR2268539.

R. Basu, V. Sidoravicius and A. Sly. Last passage percolation with a defect line and the solution of the slow bond problem. ArXiv Mathematics e-prints (2016). arXiv: $1408.3464 v 3$.

A. Borodin and P. L. Ferrari. Large time asymptotics of growth models on spacelike paths. I. PushASEP. Electron. J. Probab. 13, no. 50, 1380-1418 (2008). MR2438811.

A. Borodin, P. L. Ferrari and T. Sasamoto. Transition between Airy 1 and Airy $_{2}$ processes and TASEP fluctuations. Comm. Pure Appl. Math.61 (11), 1603-1629 (2008). MR2444377.

A. Borodin and S. Péché. Airy kernel with two sets of parameters in directed percolation and random matrix theory. J. Stat. Phys. 132 (2), 275-290 (2008). MR2415103.

E. Cator and L. P. R. Pimentel. On the local fluctuations of last-passage percolation models. Stochastic Process. Appl. 125 (2), 538-551 (2015). MR3293293.

I. Corwin, P. L. Ferrari and S. Péché. Universality of slow decorrelation in KPZ growth. Ann. Inst. Henri Poincaré Probab. Stat. 48 (1), 134-150 (2012). MR2919201.

P. L. Ferrari, P. Ghosal and P. Nejjar. Limit law of a second class particle with non-random initial data $(2018+)$. To appear in Ann. Inst. H. Poincaré Probab. Statist.

P. L. Ferrari and P. Nejjar. Anomalous shock fluctuations in TASEP and last passage percolation models. Probab. Theory Related Fields 161 (1-2), 61-109 (2015a). MR3304747.

P. L. Ferrari and P. Nejjar. Shock fluctuations in flat TASEP under critical scaling. J. Stat. Phys. 160 (4), 985-1004 (2015b). MR3373648.

P. L. Ferrari and A. Occelli. Universality of the GOE Tracy-Widom distribution for TASEP with arbitrary particle density. Electron. J. Probab. 23, Paper No. 51, 24 (2018). MR3814245.

P. L. Ferrari and H. Spohn. On time correlations for KPZ growth in one dimension. SIGMA Symmetry Integrability Geom. Methods Appl. 12, Paper No. 074, 23 (2016). MR3529743.

K. Johansson. Shape fluctuations and random matrices. Comm. Math. Phys. 209 (2), 437-476 (2000a). MR1737991.

K. Johansson. Transversal fluctuations for increasing subsequences on the plane. Probab. Theory Related Fields 116 (4), 445-456 (2000b). MR1757595.

K. Johansson. Two time distribution in Brownian directed percolation. Comm. Math. Phys. 351 (2), 441-492 (2017). MR3613511.

K. Johansson. The two-time distribution in geometric last-passage percolation. ArXiv Mathematics e-prints (2018). arXiv: 1802.00729.

T. M. Liggett. Interacting particle systems, volume 276 of Grundlehren der Mathematischen Wissenschaften [Fundamental Principles of Mathematical Sciences]. Springer-Verlag, New York (1985). ISBN 0-387-96069-4. MR776231.

L. P. R. Pimentel. Local behavior of Airy processes. ArXiv Mathematics e-prints (2017). arXiv: 1704.01903. 
J. Quastel and M. Rahman. TASEP fluctuations with soft-shock initial data. ArXiv Mathematics e-prints (2018). arXiv: 1801.06143.

G. Shinault and C. A. Tracy. Asymptotics for the Covariance of the Airy 2 process. J. Stat. Phys. 143 (1), 60-71 (2011). MR2787973.

H. Widom. On Asymptotics for the Airy process. J. Statist. Phys. 115 (3-4), 1129-1134 (2004). MR2054175. 